\title{
Fake superpotential for large and small extremal black holes
}

\author{
L. Andrianopoli, ${ }^{a, b}$ R. D’Auria, ${ }^{a, b}$ S. Ferrara ${ }^{c, d}$ and M. Trigiante ${ }^{a, b}$ \\ ${ }^{a}$ Dipartimento di Fisica, Politecnico di Torino, \\ Corso Duca degli Abruzzi 24, I-10129 Turin, Italy \\ ${ }^{b}$ INFN Sezione di Torino, \\ Via Pietro Giuria, 1, I-10125 Turin, Italy \\ ${ }^{c}$ Physics Department, Theory Unit, CERN, \\ CH 1211, Geneva 23, Switzerland \\ ${ }^{d}$ INFN - Laboratori Nazionali di Frascati, \\ Via E. Fermi 40, 00044 Frascati, Italy \\ E-mail: laura.andrianopoli@polito.it, riccardo.dauria@polito.it, \\ sergio.ferrara@cern.ch, mario.trigiante@polito.it
}

ABSTRACT: We consider the fist order, gradient-flow, description of the scalar fields coupled to spherically symmetric, asymptotically flat black holes in extended supergravities. Using the identification of the fake superpotential with Hamilton's characteristic function we clarify some of its general properties, showing in particular (besides reviewing the issue of its duality invariance) that $W$ has the properties of a Liapunov's function, which implies that its extrema (associated with the horizon of extremal black holes) are asymptotically stable equilibrium points of the corresponding first order dynamical system (in the sense of Liapunov). Moreover, we show that the fake superpotential $W$ has, along the entire radial flow, the same flat directions which exist at the attractor point. This allows to study properties of the ADM mass also for small black holes where in fact $W$ has no critical points at finite distance in moduli space. In particular the $W$ function for small non-BPS black holes can always be computed analytically, unlike for the large black-hole case.

KeYwords: Black Holes, Supersymmetry and Duality, Extended Supersymmetry, Supergravity Models 


\section{Contents}

1 Introduction 1

2 Some general properties of the $w$ function $\quad 3$

$2.1 I_{4}$ invariant for $\mathcal{N}=2,4,8$ supergravities $\quad 6$

2.2 The $W$ function and duality 8

2.3 The issue of stability: asymptotic stability of the critical points 9

$\begin{array}{ll}2.4 & \text { The issue of flat directions } \\ \end{array}$

3 Small black holes in the $\mathcal{N}=8$ theory $\quad 19$

4 Small black holes in the $\mathcal{N}=4$ theory 20

$\begin{array}{lll}4.1 & \text { Large orbits } & 22\end{array}$

$\begin{array}{lll}4.2 & \text { Small orbits } & 22\end{array}$

4.3 The $\mathcal{W}$ function for small orbits $\quad 23$

4.4 Small versus large orbits $\quad 24$

5 Small black holes in $\mathcal{N}=\mathbf{2}$ magic models $\quad \mathbf{2 7}$

$\begin{array}{ll}5.1 D=6 \text { uplift of magic models } & 27\end{array}$

$\begin{array}{ll}5.2 D=5 \text { uplift } & 27\end{array}$

$\begin{array}{ll}5.3 D & D=4 \text { analysis }\end{array} 28$

5.4 ADM mass for small black holes at $D=4 \quad 28$

5.5 The $W$ function of the small black holes in the $t^{3}$-Model 28

$\begin{array}{ll}\text { A Proof of eq. (2.12) } & 28\end{array}$

B Stability and asymptotic stability in the sense of Liapunov 30

C Properties of the Vielbein on $\mathscr{M}_{\text {scal }} \quad 31$

D An explicit parametrization of the $\mathcal{N}=4$ scalar manifold 31

\section{Introduction}

It is well known that in $N$-extended supergravity based on symmetric coset manifolds $G / H$ the dynamics of extremal (spherically symmetric, asymptotically flat) black holes is encoded in a "fake" superpotential function $W[1-6]^{1}$ which, for large black holes, is entirely specified by the duality orbit [8] of the dyonic charge vector $\mathcal{P}=\left(p^{\Lambda}, q_{\Lambda}\right)\left(\Lambda=1, \cdots, n_{V}\right)$ and the asymptotic values at radial infinity of the scalars of the theory: $\left(\phi_{0}^{r}\right) \in G / H$.

\footnotetext{
${ }^{1}$ The idea of "fake" supersymmetry was first introduced in [7].
} 
It has not been appreciated enough that many properties of the $W$ function are not only true at the horizon of regular extremal black holes, defined by critical points $\phi_{*}$ of $W$ :

$$
\left.\frac{\partial W}{\partial \phi^{r}}\right|_{\phi^{r}=\phi_{*}^{r}}=0,
$$

where space-time is $A d S_{2} \times S_{2}$, but in fact they are valid on the entire radial flow and in particular at spatial infinity where space-time is flat. All these properties naturally follow from the identification of the $W$-function with Hamilton's characteristic function $[3,10,11]$ of an autonomous Hamiltonian system. ${ }^{2}$ In [3], the fact that the radial evolution of scalar fields and the metric of spherically symmetric, asymptotically flat solutions, is described by an autonomous Hamiltonian system, was used to show that the problem of defining a first order description of black holes in terms of a superpotential $W$ is equivalent to a HamiltonJacobi problem: The $W$ function is a solution to the Hamilton-Jacobi equation associated with the Hamiltonian system and defines a system of first order gradient-flow equations for the scalar fields. From the identification of the superpotential $W$ with Hamilton's characteristic function, some important general properties follow:

- $W$ is a positive definite function on the moduli space;

- The derivative of $W$ along the flow, moving from the horizon to radial infinity, is always positive;

- $W$ for extremal solutions is duality invariant.

The latter property was originally conjectured in [2] and later proven in [3]. Eventually, in [4-6] the explicit construction of $W$ in terms of duality invariants was completed.

As we shall prove, the above properties of $W$, in the presence of a critical point $\phi_{*}$ of the first order system, promote $W$ to a Liapunov's function (see for instance $[19,20]$ ), allowing to make precise statements about the asymptotic stability of $\phi_{*}$ (namely that $\phi_{*}$ is not just an attractive equilibrium point, but also stable), with no need of computing the Hessian of the potential. The existence of $W$, even in a neighborhood of the critical point, provides an alternative (and more powerful) characterization of its attractiveness and stability properties.

Of particular interest are orbits of extremal, large, black holes ${ }^{3}$ in which the critical points are not isolated. This feature is related, in the symmetric models, to the existence of flat directions of the scalar potential $V$ [21]. We shall prove in full generality, using the duality invariance of $W$, that $W$ and $V$ have the same symmetry properties, and thus that they also have the same flat directions. These flat directions, which have an intrinsic group theoretical characterization in terms of the stabilizer of the duality orbit of the quantized charges $[8,9]$, are also a feature of the central and matter charges.

\footnotetext{
${ }^{2}$ See also [12-18] for related independent works.

${ }^{3}$ Large black holes are solutions for which a certain (quartic) duality-invariant expression of the charge vector $\mathcal{P}$, called $I_{4}(\mathcal{P})$ does not vanish. For small black holes, on the other hand, $I_{4}(\mathcal{P})=0$. A definition of $I_{4}(\mathcal{P})$, and its $G$-invariant form for symmetric geometries $G / H$ is summarized in subsection 2.1.
} 
An other consequence of the general properties of $W$ is that the functional form of $W\left(i_{n}, I_{4}\right)$, where $i_{n}$ are the $\mathrm{H}$-invariant combinations of the moduli $\phi^{r}$ and charges $\mathcal{P}$, can also be calculated for $I_{4}=0$, in which case the classical horizon area vanishes and eq. (1.1) has no solutions (in the interior of the moduli space). More precisely, for $I_{4}=0$, eq. (1.1) has a runaway solution $W=0$ at the boundary of the moduli space where some $\phi^{r} \rightarrow \infty[22,23]$.

It is the aim of this note to further specify general properties of the $W$ function for large and small black holes, such as their moduli spaces and symmetries. Moreover, depending on the number $N$ of supersymmetries, $W\left(I_{4}=0\right)$ can be obtained by a suitable limit of large black hole solutions (where $I_{4} \neq 0$ ), in such a way that $W$ is always given by a calculable algebraic function of the $H$-invariants. The way the limit is performed also allows us to simply understand the interplay of BPS properties of small black holes versus large solutions.

As a byproduct, our analysis shows that $I_{4}=0$ black holes in $\mathcal{N}=8$ are always BPS (distinct in three orbits with different fractions of supersymmetry), while in $\mathcal{N}=4,2$ theories $I_{4}=0$ black holes can be non-BPS. Nevertheless their $W$ function in this case can also be analytically computed from a non-BPS large black hole with vanishing central charge $\left(Z_{A B}=0, I_{4}>0\right)$.

The paper is organized as follows: In section 2 we review and outline the above mentioned properties of the $W$ superpotential. In particular we give the general form of $W$ for extremal solutions and address the issue of asymptotic stability of the critical points on $W$, by showing that $W$ can be identified with a Liapunov's function. We also prove that the potential $V$, the superpotential $W$, together with the central and matter charges, have the same flat directions defined by the $G$-orbit of the quantized charges $\mathcal{P}$. In sections $3,4,5$ we analyze small black holes for $\mathcal{N}=8,4$ and 2 respectively. Three appendices containing derivations of properties of the $W$-function discussed in the text, together with other mathematical details, are included.

\section{Some general properties of the $w$ function}

Let us review some general properties of the fake superpotential $W$ associated with $U$ duality orbits of static, extremal, asymptotically flat black hole solutions in an extended supergravity theory with a symmetric scalar manifold $\mathscr{M}_{\text {scal }}=\frac{G}{H}$.

Let us consider an extended supergravity describing $n$ real scalar fields, spanning the manifold $\mathscr{M}_{\text {scal }}$ and $n_{V}$ vector fields $A_{\mu}^{\Lambda}$. The ansatz for the metric and the vector field strengths $F_{\mu \nu}^{\Lambda}$, for the kind of black holes we are considering, is:

$$
\begin{aligned}
d s^{2} & =-e^{2 U} d t^{2}+e^{-2 U}\left[\frac{d \tau^{2}}{\tau^{4}}+\frac{1}{\tau^{2}}\left(d \theta^{2}+\sin (\theta) d \varphi^{2}\right)\right] \\
\mathbb{F} & =\left(\begin{array}{c}
F_{\mu \nu}^{\Lambda} \\
G_{\Lambda \mu \nu}
\end{array}\right) \frac{d x^{\mu} \wedge d x^{\nu}}{2}=e^{2 U} \mathbb{C} \cdot \mathcal{M}\left(\phi^{r}\right) \cdot \mathcal{P} d t \wedge d \tau+\mathcal{P} \sin (\theta) d \theta \wedge d \varphi,
\end{aligned}
$$

where the coordinate $\tau=-1 / r$ runs from 0 , at radial infinity, to $-\infty$ at the horizon, where $e^{U(\tau)}$ vanishes. The scalar fields are taken to be functions of $\tau$ only: $\phi^{r}=\phi^{r}(\tau)$. The 
magnetic field strength $G_{\Lambda \mu \nu}$ in (2.1) is defined, as usual, as: $G_{\Lambda \mu \nu} \propto \epsilon_{\mu \nu \rho \sigma} \delta \mathcal{L} / \delta F_{\rho \sigma}^{\Lambda}, \mathcal{L}$ being the Lagrangian of the theory. The last equation in (2.1) is written in a manifestly symplectic covariant form, namely as an equality between two $2 n_{V}$ dimensional symplectic vectors, where $\mathbb{C}_{M N}, M, N=1, \ldots, 2 n_{V}$ is the $\operatorname{Sp}\left(2 n_{V}, \mathbb{R}\right)$-invariant matrix:

$$
\mathbb{C}=\left(\begin{array}{cc}
\mathbf{0} & -\mathbb{1} \\
\mathbb{1} & \mathbf{0}
\end{array}\right) \text {. }
$$

The vector $\mathcal{P} \equiv\left(p^{\Lambda}, q_{\Lambda}\right)$ consists of the quantized electric and magnetic charges. Finally the $2 n_{V} \times 2 n_{V}$ symmetric, negative defined, symplectic matrix $\mathcal{M}\left(\phi^{r}\right)_{M N} \equiv-\left(\mathbb{L} \mathbb{L}^{T}\right)_{M N}$, $\mathbb{L}\left(\phi^{r}\right)$ being the $\mathscr{M}_{\text {scal }}$ coset representative in the fundamental of $\operatorname{Sp}\left(2 n_{V}, \mathbb{R}\right)$, can also be written in the familiar form [26-28]:

$$
\mathcal{M}\left(\phi^{r}\right)=\left(\begin{array}{cc}
I+R I^{-1} R & -R I^{-1} \\
-I^{-1} R & I^{-1}
\end{array}\right),
$$

where $I_{\Lambda \Sigma} \equiv \operatorname{Im}(\mathcal{N})_{\Lambda \Sigma}<0$ is the vector kinetic matrix while $R_{\Lambda \Sigma} \equiv \operatorname{Re}(\mathcal{N})_{\Lambda \Sigma}$ defines the generalized theta-term.

Once the electric and magnetic charges of the solution are assigned, the radial evolution of the $n+1$ fields $U(\tau), \phi^{r}(\tau)$ is described by the effective action:

$$
S_{\text {eff }}=\int \mathcal{L}_{\text {eff }} d \tau=\int\left(\dot{U}^{2}+\frac{1}{2} G_{r s}(\phi) \dot{\phi}^{r} \dot{\phi}^{s}+e^{2 U} V(\phi, \mathcal{P})\right) d \tau,
$$

together with the Hamiltonian constraint, representing the extremality condition: ${ }^{4}$

$$
\mathcal{H}_{\mathrm{eff}}=\dot{U}^{2}+\frac{1}{2} G_{r s}(\phi) \dot{\phi}^{r} \dot{\phi}^{s}-e^{2 U} V(\phi, \mathcal{P})=0,
$$

the effective potential being given by $V(\phi, \mathcal{P}) \equiv-\frac{1}{2} \mathcal{P}^{T} \mathcal{M}(\phi) \mathcal{P}>0$ and the dot represents the derivative with respect to $\tau$. The radial evolution of the $n+1$ fields $U(\tau), \phi^{r}(\tau)$ in the solution admits a first order description [1-3] in terms of a fake superpotential $W(\phi, \mathcal{P})$ :

$$
\dot{U}=e^{U} W \quad, \quad \dot{\phi}^{r}=2 e^{U} G^{r s} \frac{\partial W}{\partial \phi^{s}} .
$$

If we interpret the fields $U(\tau), \phi^{r}(\tau)$ as coordinates of a Hamiltonian system in which the radial variable plays the role of time, the first order description (2.6) is equivalent to solving the Hamilton-Jacobi problem with Hamilton's characteristic function

$$
\mathcal{W}(U, \phi) \equiv 2 e^{U} W(\phi) .
$$

Indeed, in terms of $W(\phi, \mathcal{P})$, the Hamilton-Jacobi equation has the form:

$$
W^{2}+2 G^{r s} \frac{\partial W}{\partial \phi^{r}} \frac{\partial W}{\partial \phi^{s}}=V,
$$

\footnotetext{
${ }^{4}$ For non extremal black holes the value of the Hamiltonian on a solution coincides with the square of the extremality parameter. Notice that the Hamiltonian is not positive definite, being expressed as the difference of a "kinetic" and a positive "potential" term (this is in turn due to the fact that the role of the time variable is played by a spatial coordinate $\tau$ ). As a consequence of this we can have non-trivial solutions on which the Hamiltonian vanishes. These correspond to the extremal black holes.
} 
which can also be derived from the Hamiltonian constraint (2.5) using (2.6). ${ }^{5}$ We are not interested here in the most general solution to (2.8), nor to address the issue of its existence (see [3] for a discussion on this point). We are interested, instead, in the $W$ functions associated with classes of extremal solutions whose general properties are in principle known. They are completely characterized by the set of quantized charges $\mathcal{P}$ and the values of the fields at radial infinity:

$$
U(\tau=0)=0, \quad \phi^{r}(\tau=0)=\phi_{0}^{r} .
$$

We shall therefore simply denote them by: $U=U\left(\tau ; \phi_{0}\right)$ and $\phi^{r}=\phi^{r}\left(\tau ; \phi_{0}\right)$. The ADM mass and the scalar charges at infinity are given by:

$$
\begin{aligned}
M_{A D M}\left(\phi_{0}, \mathcal{P}\right) & =\dot{U}(\tau=0)=W\left(\phi_{0}, \mathcal{P}\right) \\
\Sigma^{r}\left(\phi_{0}, \mathcal{P}\right) & =\dot{\phi}^{r}(\tau=0)=2 G^{r s}\left(\phi_{0}\right) \frac{\partial W}{\partial \phi^{r}}\left(\phi_{0}, \mathcal{P}\right) .
\end{aligned}
$$

Regular (large) extremal black holes have finite horizon area $A_{H}$ and thus near the horizon $(\tau \rightarrow-\infty) e^{U}$ has the following behavior: $e^{-2 U} \sim \frac{A_{H}}{4 \pi} \tau^{2}$, where $A_{H}=A_{H}(\mathcal{P})$ is a function of the quantized charges only. In fact $\mathcal{P}$ transforms under duality (see subsection 2.2) in a symplectic representation of $G$ and $A_{H}$, as a function of $\mathcal{P}$, is expressed in terms of the quartic invariant of $G$ in this representation: $A_{H}(\mathcal{P})=4 \pi \sqrt{\left|I_{4}(\mathcal{P})\right|}$ (here we use the units $c=\hbar=G=1$, so that the Plank length is one.). Using eqs. (2.6) we see that $W$ computed on the solution evolves, in the near horizon limit, towards $\sqrt{\frac{A_{H}}{4 \pi}}$.

As far as the scalar fields are concerned, due to the attractor mechanism some of them are fixed at the horizon to values which are totally determined in terms of the quantized charges, while other scalar fields, which are flat directions of the potential, are not. That is, in the presence of flat directions, in the near horizon limit $\tau \rightarrow-\infty$ the non flat scalars evolve towards values which are totally fixed in terms of quantized charges, while the flat directions still depend, in general, on the boundary values $\phi_{0}^{r}$ taken at radial infinity $(\tau=0)$. Since only the scalars parametrizing the flat directions may depend at the horizon on $\phi_{0}^{r}$, the near horizon geometry, which is determined in terms of the potential, will only depend on the quantized charges, consistently with the attractor mechanism. Summarizing, for large black holes, we have:

$$
\begin{aligned}
\lim _{\tau \rightarrow-\infty} e^{-2 U} & =\sqrt{\left|I_{4}(\mathcal{P})\right|} \tau^{2}, \quad \lim _{\tau \rightarrow-\infty} \phi^{r}(\tau)=\phi_{*}^{r}, \\
\lim _{\tau \rightarrow-\infty} W^{2}\left(\phi\left(\tau ; \phi_{0}\right), \mathcal{P}\right) & =W^{2}\left(\phi_{*}, \mathcal{P}\right)=V\left(\phi_{*}, \mathcal{P}\right)=\sqrt{\left|I_{4}(\mathcal{P})\right|} .
\end{aligned}
$$

Small black holes are characterized by vanishing horizon area, i.e. by quantized charges for which $I_{4}(\mathcal{P})=0$. For $\tau \rightarrow-\infty$ the warp factor has the following behavior: $e^{-2 U} \sim \tau^{\alpha}$, $\alpha<2$. In the same limit scalar fields typically flow to values which are at the boundary

\footnotetext{
${ }^{5}$ In the case of non-extremal solutions the Hamilton-Jacobi equation reads $\left(\frac{\partial \mathcal{W}}{\partial U}\right)^{2}+2 G^{r s}(\phi) \frac{\partial \mathcal{W}}{\partial \phi^{r}} \frac{\partial \mathcal{W}}{\partial \phi^{s}}=$ $4 e^{2 U} V+4 c^{2}$, and the corresponding first order equations have the form $\dot{U}=\frac{1}{2} \frac{\partial \mathcal{W}}{\partial U}, \dot{\phi}^{r}=G^{r s}(\phi) \frac{\partial \mathcal{W}}{\partial \phi^{s}}$. If $c \neq 0$ however, as it is apparent from the Hamilton-Jacobi equation, the dynamical system can have no equilibrium point $\frac{\partial \mathcal{W}}{\partial U}=\frac{\partial \mathcal{W}}{\partial \phi^{r}}=0$.
} 
of the scalar manifold. Either for large or for small solutions, from the first of (2.6) we deduce the following boundary condition for $W$ :

$$
\lim _{\tau \rightarrow-\infty} e^{U\left(\tau ; \phi_{0}\right)} W\left(\phi\left(\tau ; \phi_{0}\right), \mathcal{P}\right)=\lim _{\tau \rightarrow-\infty} \dot{U}=0 .
$$

This allows us to write $W(\phi, \mathcal{P})$ for the two kinds of solutions in the following form (see [3]):

$$
W\left(\phi_{0}, \mathcal{P}\right)=\int_{-\infty}^{0} e^{2 U\left(\tau ; \phi_{0}\right)} V\left(\phi\left(\tau ; \phi_{0}\right), \mathcal{P}\right) d \tau
$$

It should be stressed that the above expression allows to write the $W$ function for a given class of solutions as a free function of the point $\phi_{0}$ on the scalar manifold and of the quantized charges: Given a charge vector $\mathcal{P}$ and a point $\phi_{0}=\left(\phi_{0}^{r}\right)$ in $\mathscr{M}_{\text {scal }}$, the corresponding value of $W$ is given by the integral over $\tau$ of $e^{2 U} V$, computed along the unique solution originating at infinity in $\phi_{0}$.

\section{$2.1 \quad I_{4}$ invariant for $\mathcal{N}=2,4,8$ supergravities}

In $\mathcal{N}>2$ theories and in $\mathcal{N}=2$ theories based on symmetric spaces for the (vector multiplet) scalar fields, the entropy area law reads (the Boltzmann constant $k_{B}$ being one in our units):

$$
S=\frac{A_{H}}{4}=\pi \sqrt{\left|I_{4}(\mathcal{P})\right|},
$$

where, as anticipated in the previous section, $I_{4}(\mathcal{P})$ is a certain quartic invariant of the dyonic charge vector $\mathcal{P}$ and depends on the particular theory under consideration. Since $I_{4}(\mathcal{P})$ is moduli-independent, it can be expressed either in terms of the quantized charges $\mathcal{P}$ or in terms of the (dressed) central and matter charges $Z_{A B}(\phi, \mathcal{P}), Z_{I}(\phi, \mathcal{P})$ (see subsection 2.3 for a precise definition of the latter). For our convenience we recall here the actual form of $I_{4}(\mathcal{P})$ in terms of the central and matter charges.

For $\mathcal{N}=2$ theories, based on special geometry, we can define five $H$-invariant quantities $i_{n}$, as follows [4]:

$$
\begin{aligned}
i_{1} & \equiv Z \bar{Z}, \\
i_{2} & \equiv g^{i \bar{\jmath}} Z_{i} \bar{Z}_{\bar{\jmath}}, \\
i_{3} & \equiv \frac{1}{3} \operatorname{Re}\left(Z N_{3}\left(\bar{Z}_{\bar{\imath}}\right)\right), \\
i_{4} & \equiv-\frac{1}{3} \operatorname{Im}\left(Z N_{3}\left(\bar{Z}_{\bar{\imath}}\right)\right), \\
i_{5} & \equiv g^{i \bar{\imath}} C_{i j k} \bar{C}_{\bar{\imath} \bar{\jmath} \bar{k}} \bar{Z}^{j} \bar{Z}^{k} Z^{\bar{\jmath}} Z^{\bar{k}},
\end{aligned}
$$

where $N_{3}\left(\bar{Z}_{\bar{\imath}}\right) \equiv C_{i j k} \bar{Z}^{i} \bar{Z}^{j} \bar{Z}^{k}, Z_{i} \equiv D_{i} Z$ and $Z^{\bar{\imath}} \equiv g^{\bar{i} i} Z_{i}$. In terms of these quantities the quartic invariant reads:

$$
I_{4}=\left(i_{1}-i_{2}\right)^{2}+4 i_{4}-i_{5}=I_{4}(\mathcal{P}),
$$


where, as anticipated in the previous subsection, $\mathcal{P}$ transforms in a symplectic representation of $G$ and $I_{4}(\mathcal{P})$ is the only non-vanishing invariant quantity built out of the charge vector. Note that, for the quadratic series $\left(C_{i j k}=0\right)$ we have: $I_{4}=I_{2}^{2}$, where $I_{2} \equiv\left|i_{1}-i_{2}\right|$.

For $\mathcal{N}=4$, we can define two $\mathrm{SU}(4) \times \mathrm{SO}(\mathrm{n})$ invariants:

$$
\begin{aligned}
S_{1} & \equiv \frac{1}{2} Z_{A B} \bar{Z}^{A B}-Z_{I} \bar{Z}_{\bar{J}} \delta^{I \bar{J}}, \\
S_{2} & \equiv \frac{1}{4} \epsilon^{A B C D} Z_{A B} Z_{C D}-Z_{I} Z_{J} \delta^{I J},
\end{aligned}
$$

in terms of the central charges $Z_{A B}=-Z_{B A}, A, B=1, \ldots, 4$, and the $n$ matter charges $Z_{I}, I=1, \ldots, n$. Then the unique quartic $G=\mathrm{SL}(2, \mathbb{R}) \times \mathrm{SO}(6, \mathrm{n})$-invariant reads:

$$
I_{4}^{(\mathcal{N}=4)}(\mathcal{P}) \equiv S_{1}^{2}-\left|S_{2}\right|^{2}
$$

and the black hole potential is:

$$
V^{(\mathcal{N}=4)}(\phi, \mathcal{P})=\frac{1}{2} Z_{A B} \bar{Z}^{A B}+Z_{I} \bar{Z}^{I}
$$

Finally, in the $\mathcal{N}=8$ theory the Cartan $G=\mathrm{E}_{7(7)}$-quartic invariant is given by the expression [30]:

$$
I_{4}^{(\mathcal{N}=8)}(\mathcal{P}) \equiv \operatorname{Tr}\left[\left(\mathbb{Z} \mathbb{Z}^{\dagger}\right)^{2}\right]-\left[\operatorname{Tr}\left(\mathbb{Z} \mathbb{Z}^{\dagger}\right)\right]^{2}+8 \operatorname{Re}[\operatorname{Pf}(\mathbb{Z})]
$$

where $\mathbb{Z} \equiv\left(Z_{A B}\right)=-\mathbb{Z}^{T}, A, B=1, \ldots, 8$, is the complex central charge matrix [31]. In terms of the four skew-eigenvalues $z_{i}, i=1, \ldots, 4$, of $Z_{A B}, I_{4}^{(\mathcal{N}=8)}$ reads:

$$
I_{4}^{(\mathcal{N}=8)}(\mathcal{P}) \equiv \sum_{i=1}^{4}\left|z_{i}\right|^{4}-2 \sum_{i<j}\left|z_{i}\right|^{2}\left|z_{j}\right|^{2}+4\left(z_{1} z_{2} z_{3} z_{4}+\bar{z}_{1} \bar{z}_{2} \bar{z}_{3} \bar{z}_{4}\right) \text {. }
$$

The black hole effective potential has the following form:

$$
V^{(\mathcal{N}=8)}(\phi, \mathcal{P})=\frac{1}{2} Z_{A B} \bar{Z}^{A B}=\sum_{i=1}^{4}\left|z_{i}\right|^{2} .
$$

In any extended supergravity, BPS solutions are described by $W=\left|z_{h}\right|$, where $z_{h}$ is the highest skew-eigenvalue (i.e. eigenvalue with highest modulus) of the central charge matrix $Z_{A B}$ (for $\mathcal{N}=2, Z_{A B}=Z \epsilon_{A B}, A, B=1,2$, and $z_{h}=Z$ ). Therefore it is also true that:

$$
V=\left|z_{h}\right|^{2}+2 G^{r s} \partial_{r}\left|z_{h}\right| \partial_{s}\left|z_{h}\right| \text {. }
$$

If however $\mathcal{P}$ is not in a BPS orbit, the flow defined by $W=\left|z_{h}\right|$ does not correspond to a physically acceptable solution and a different $W$-function should be used.

In particular, in the $\mathcal{N}=8$ case for non-BPS configurations the corresponding $W$ function satisfies the following inequalities:

$$
\left|z_{h}\right|^{2}<W^{2} \leq 4\left|z_{h}\right|^{2}
$$

the lower bound being saturated only for BPS solutions. The upper bound originates from the general property: $W^{2} \leq V \leq 4\left|z_{h}\right|^{2}$. For non-BPS large black holes, it can be proven that, at the attractor point, $\left|z_{i}\right|=\rho=\left|z_{h}\right|$ and the upper bound is saturated: $W=2 \rho$. 


\subsection{The $W$ function and duality}

It is known that the on-shell global symmetries of an extended supergravity, at the classical level, are encoded in the isometry group $G$ of the scalar manifold (if non-empty), whose action on the scalar fields is associated with a simultaneous linear symplectic action on the field strengths $F^{\Lambda}$ and their duals $G_{\Lambda}$. This duality action of $G$ is defined by a symplectic representation $D$ of $G$ :

$$
g \in G:\left\{\begin{array}{l}
\phi^{r} \rightarrow \phi^{r \prime}=g \star \phi^{r} \\
\left(\begin{array}{c}
F^{\Lambda} \\
G_{\Lambda}
\end{array}\right) \rightarrow D(g) \cdot\left(\begin{array}{c}
F^{\Lambda} \\
G_{\Lambda}
\end{array}\right),
\end{array}\right.
$$

where $g \star$ denotes the non-linear action of $g$ on the scalar fields and $D(g)$ is the $2 n_{v} \times 2 n_{v}$ symplectic matrix associated with $g$. The matrix $\mathcal{M}(\phi)$ transforms under $G$ as follows:

$$
\mathcal{M}(g \star \phi)=D(g)^{-T} \mathcal{M}(\phi) D(g)^{-1} .
$$

A duality transformation $g \in G$ maps a black hole solution $U(\tau), \phi^{r}(\tau)$ with charges $\mathcal{P}$ into a new solution $U^{\prime}(\tau)=U(\tau), \phi^{\prime r}(\tau)=g \star \phi^{r}(\tau)$ with charges $\mathcal{P}^{\prime}=D(g) \mathcal{P}$. More specifically, if $U(\tau), \phi^{r}(\tau)$ is defined by the boundary condition $\phi_{0}$ for the scalar fields, $U^{\prime}(\tau)=U(\tau), \phi^{\prime r}(\tau)$ is the unique solution, within our class, with charges $\mathcal{P}^{\prime}$ defined by the boundary condition $\phi_{0}^{\prime}=g \star \phi_{0}$

$$
g \in G:\left\{\begin{array} { l } 
{ U ( \tau ; \phi _ { 0 } ) } \\
{ \phi ( \tau ; \phi _ { 0 } ) } \\
{ \mathcal { P } }
\end{array} \longrightarrow \left\{\begin{array}{l}
U^{\prime}\left(\tau ; g \star \phi_{0}\right)=U\left(\tau ; \phi_{0}\right) \\
\phi^{\prime}\left(\tau ; g \star \phi_{0}\right)=g \star \phi\left(\tau ; \phi_{0}\right) \\
\mathcal{P}^{\prime}=D(g) \mathcal{P}
\end{array}\right.\right.
$$

Using eqs. (2.23) and (2.24), we see that the effective potential is invariant if we act on $\phi^{r}$ and $\mathcal{P}$ by means of $G$ simultaneously:

$$
V(\phi, \mathcal{P})=V(g \star \phi, D(g) \mathcal{P}) .
$$

This implies that $V$, as a function of the scalar fields and quantized charges, is $G$-invariant. From this property of $V$ it follows that the effective action (2.4) and the extremality constraint (2.5) are manifestly duality invariant. Let us show now that the $W$ function shares with $V$ the same symmetry property (2.25), namely that it is $G$-invariant as well:

$$
W(\phi, \mathcal{P})=W(g \star \phi, D(g) \mathcal{P}) .
$$

This is easily shown using the general form (2.12) and eqs. (2.24):

$$
\begin{aligned}
W\left(g \star \phi_{0}, D(g) \mathcal{P}\right) & =\int_{-\infty}^{0} e^{2 U^{\prime}\left(\tau ; g \star \phi_{0}\right)} V\left(\phi^{\prime}\left(\tau ; g \star \phi_{0}\right), D(g) \mathcal{P}\right) d \tau= \\
& =\int_{-\infty}^{0} e^{2 U\left(\tau ; \phi_{0}\right)} V\left(g \star \phi\left(\tau ; \phi_{0}\right), D(g) \mathcal{P}\right) d \tau= \\
& =\int_{-\infty}^{0} e^{2 U\left(\tau ; \phi_{0}\right)} V\left(\phi\left(\tau ; \phi_{0}\right), \mathcal{P}\right) d \tau=W\left(\phi_{0}, \mathcal{P}\right) .
\end{aligned}
$$


Being the ADM mass expressed in terms of $W$, see eq. (2.10), it is a $G$-invariant quantity as well:

$$
M_{A D M}\left(\phi_{0}, \mathcal{P}\right)=M_{A D M}\left(g \star \phi_{0}, D(g) \mathcal{P}\right) .
$$

Extremal black-holes can be grouped into orbits with respect to the duality action (2.24) of $G$. These orbits are characterized in terms of $G$-invariant functions of the scalar fields and the quantized charges, which are expressed in terms of $H$-invariant functions of the central and matter charges. One of these is the scalar-independent quartic invariant $I_{4}(\mathcal{P})$ of $G$ which defines the area of the horizon for large black holes. Small black holes, on the other hand, belong to the orbits in which $I_{4}(\mathcal{P})=0$.

\subsection{The issue of stability: asymptotic stability of the critical points}

Let us notice, from eq. (2.12), that $W$ is always positive definite, since the effective potential is. Moreover its derivative along the solution $\phi^{r}(\tau)$ is positive definite as well (except in $\phi_{*}$ where it vanishes):

$$
\frac{d W}{d \tau}=\dot{\phi}^{r} \partial_{r} W=e^{-U} G_{r s}(\phi) \dot{\phi}^{r} \dot{\phi}^{s}>0
$$

We see that, if $\phi_{*}$ is isolated, $W$ has the properties of a Liapunov's function and thus, in virtue of Liapunov's theorem, $\phi_{*}$ is a stable attractor point (we refer the reader to appendix B for a brief review of the notion of asymptotic stability in the sense of Liapunov and of Liapunov's theorem, see also standard books like $[19,20]$ ). This conclusion extends to models based on a generic (not necessarily homogeneous) scalar manifold: The very existence of the $W$-function (i.e. of a solution to the Hamilton-Jacobi equation) even just in a neighborhood of an isolated critical point $\phi_{*}$ is enough to guarantee asymptotic stability of $\phi_{*}$, and thus that the horizon is a stable attractor. Let us emphasize that in this case we need not evaluate the Hessian of the potential on $\phi_{*}$. In other words the (local) existence of $W$ can be taken as an alternative and more powerful characterization of the attractiveness and stability properties of the horizon point $\phi_{*}$.

There is a class of large extremal solutions, however, in which the critical points, defining the near-horizon behavior of the scalar fields, are not isolated but rather span a hypersurface $\mathcal{C}$ of the scalar manifold. This is the case of the non-BPS solutions with $I_{4}<0$ in the symmetric models. As we are going to show below, in full generality, the existence of this locus of critical points is related to the existence of $n_{f}<n$ flat directions $\varphi^{\alpha}, \alpha=1, \ldots, n_{f}$, of both the scalar potential $V$ and the $W$ function. The critical hypersurface $\mathcal{C}$ has in this case dimension $n_{f}$ and is spanned by $\left(\varphi^{\alpha}\right)$. As far as the global behavior of the flows is concerned, the analysis of the simple STU model (see [29] for a discussion on this point) suggests a general property: The scalar manifold can be decomposed in hypersurfaces $\mathscr{M}_{(\alpha)}$ of dimension $n-n_{f}$ which intersect the hypersurface of critical points $\mathcal{C}$ in a single point $\left.\phi_{*}\right|_{\alpha}$ characterized by fixed values $\varphi^{\alpha}$ of the flat directions. The hypersurfaces $\mathscr{M}_{(\alpha)}$ have the property of being invariant with respect to the flow, namely that, choosing the initial point $\phi_{0}$ on a given $\mathscr{M}_{\alpha}$, the entire flow will be contained within the same hypersurface. Within each $\mathscr{M}_{(\alpha)}$ the critical point $\left.\phi_{*}\right|_{\alpha}$ is isolated 
and Liapunov's theorem applies, implying it is asymptotically stable or, equivalently, a stable attractor.

\subsection{The issue of flat directions}

Let us denote by $G_{0} \subset G$ the little group (or stabilizer) of the orbit of the quantized charges $\mathcal{P}$ under the action of $G[8,9,24]$ :

$$
g_{0} \in G_{0}: \quad D\left(g_{0}\right) \mathcal{P}=\mathcal{P} .
$$

Of course the embedding of $G_{0}$ within $G$ depends in general on $\mathcal{P}$. Let us show that the scalar fields $\varphi^{\alpha}$ spanning the submanifold $G_{0} / H_{0}, H_{0}$ being the maximal compact subgroup of $G_{0}$, are flat directions of the potential and of the $W$-function, namely that neither $V$ nor $W$, depend on $\varphi^{\alpha}$. Since we are interested in the part of the little group which has a free action on the moduli, we shall define $G_{0}$ modulo compact group-factors. For instance if the little group is $\mathrm{SU}(3) \times \mathrm{SU}(2,1)$, we define $G_{0}$ to be $\mathrm{SU}(2,1)$ and thus $H_{0}=\mathrm{U}(2)$. For a summary of the orbits of regular extremal black holes in the various theories and of the corresponding moduli spaces $G_{0} / H_{0}$ see table 1 .

To prove that $\varphi^{\alpha}$ are flat directions of both $V$ and $W$, let us decompose the $n$ scalar fields $\phi^{r}$ into the $\varphi^{\alpha}$ scalars parametrizing the submanifold $G_{0} / H_{0}$ and scalars $\varphi^{k}$, which can be chosen to transform linearly with respect to $H_{0}$. Let us stress at this point that the coordinates $\varphi^{\alpha}, \varphi^{k}$ will in general depend on the original ones $\phi^{r}$ and on the electric and magnetic charges, namely:

$$
\varphi^{\alpha}=\varphi^{\alpha}\left(\phi^{r}, p^{\Lambda}, q_{\Lambda}\right), \quad \varphi^{k}=\varphi^{k}\left(\phi^{r}, p^{\Lambda}, q_{\Lambda}\right) .
$$

Let us choose, for convenience, a basis of coordinates in the moduli space such that the first $n_{f}$ components of $\phi^{r}$ coincide with the $\varphi^{\alpha}$, the others being $\varphi^{k}$, that is $\phi^{\alpha}=\varphi^{\alpha}$, $\phi^{k}=\varphi^{k}$. We can move along the $\phi^{\alpha}$ direction through the action of isometries in $G_{0}$. We shall consider infinitesimal isometries in $G_{0}$ whose effect is to shift the $\alpha$-scalars only:

$$
g_{0} \in G_{0}: \phi^{r} \rightarrow\left(g_{0} \star \phi\right)^{r}=\phi^{r}+\delta_{\alpha}^{r} \delta \phi^{\alpha} \quad, \quad \mathcal{P} \rightarrow \mathcal{P}^{\prime}=\mathcal{P}+\delta \mathcal{P}=\mathcal{P},
$$

where we have used the definition of $G_{0},(2.30)$. Let us now use eqs. (2.25) and (2.26) to evaluate the corresponding infinitesimal variations of $V$ and $W$ :

$$
\begin{aligned}
V\left(\phi^{r}, \mathcal{P}\right) & =V\left(\phi^{r}+\delta \phi^{r}, \mathcal{P}+\delta \mathcal{P}\right)=V\left(\phi^{k}, \phi^{\alpha}+\delta \phi^{\alpha}, \mathcal{P}\right) . \\
W\left(\phi^{r}, \mathcal{P}\right) & =W\left(\phi^{r}+\delta \phi^{r}, \mathcal{P}+\delta \mathcal{P}\right)=W\left(\phi^{k}, \phi^{\alpha}+\delta \phi^{\alpha}, \mathcal{P}\right) .
\end{aligned}
$$

We conclude that $\frac{\partial V}{\partial \phi^{\alpha}}=\frac{\partial W}{\partial \phi^{\alpha}}=0$, namely that $\phi^{\alpha}$ are flat direction of both functions. Using eq. (2.10) we see that the same property holds for the ADM mass: $\frac{\partial}{\partial \phi^{\alpha}} M_{A D M}=0$. Let us now give a general characterization of the $W$-function in terms of the central and matter charges. We can write the coset representative $\mathbb{L}\left(\phi^{r}\right)$ of $\mathscr{M}_{\text {scal }}$ as the product of the $G_{0} / H_{0}$ coset representative $\mathbb{L}_{0}\left(\phi^{\alpha}\right)$ times a matrix $\mathbb{L}_{1}\left(\phi^{k}\right)$ depending on the remaining scalars:

$$
\mathbb{L}\left(\phi^{r}\right)=\mathbb{L}\left(\phi^{\alpha}, \phi^{k}\right)=\mathbb{L}_{0}\left(\phi^{\alpha}\right) \mathbb{L}_{1}\left(\phi^{k}\right) .
$$




\begin{tabular}{|c|c|c|c|c|c|}
\hline $\mathcal{N}$ & $\frac{G}{H}$ & orbit & $\frac{G_{0}}{H_{0}}$ & $\mathbf{R}_{0}$ & $\mathbf{R}_{1}$ \\
\hline 8 & $\frac{E_{7(7)}}{\operatorname{SU}(8)}$ & $\begin{array}{c}\text { I } \\
\text { III }\end{array}$ & $\begin{array}{c}\mathrm{E}_{6(2)} \\
\mathrm{SU}(2) \times \mathrm{SU}(6) \\
\frac{\mathrm{E}_{6(6)}}{\mathrm{USp}(8)} \\
\end{array}$ & $\begin{array}{c}(2,20) \\
42 \\
\end{array}$ & $\begin{array}{c}(\mathbf{1}, \mathbf{1 5})+\text { c.r. } \\
\mathbf{1}+\mathbf{2 7}\end{array}$ \\
\hline 6 & $\frac{\mathrm{SO}^{*}(12)}{\mathrm{U}(6)}$ & $\begin{array}{c}\text { I } \\
\text { II } \\
\text { III }\end{array}$ & $\begin{array}{c}\frac{\mathrm{SU}(4,2)}{\mathrm{S}[\mathrm{U}(4) \times \mathrm{U}(2)]} \\
- \\
\frac{\mathrm{SU}^{*}(6)}{\mathrm{USp}(6)} \\
\end{array}$ & $\begin{array}{c}(4,2)_{-3}+\text { c.r. } \\
14 \\
\end{array}$ & $\begin{array}{c}(\mathbf{6}, \mathbf{1})_{+2}+(\mathbf{1}, \mathbf{1})_{-4}+c . r . \\
2 \times \mathbf{1}+\mathbf{1 4}\end{array}$ \\
\hline 5 & $\frac{\mathrm{SU}(5,1)}{\mathrm{U}(5)}$ & I & $\frac{\mathrm{SU}(2,1)}{\mathrm{U}(2)}$ & $\mathbf{2}_{+3}+$ c.r. & $3 \times \mathbf{1}_{-2}+$ c.r. \\
\hline 4 & $\begin{array}{c}\frac{\mathrm{SL}(2, \mathbb{R})}{\mathrm{SO}(2)} \times \\
\times \frac{\mathrm{SO}(6, n)}{\mathrm{SO}(6) \times \mathrm{SO}(n)}\end{array}$ & III & $\begin{array}{c}\frac{\mathrm{SO}(4, n)}{\mathrm{SO}(4) \times \mathrm{SO}(n)} \\
\frac{\mathrm{SO}(6, n-2)}{\mathrm{SO}(6) \times \mathrm{SO}(n-2)} \\
\mathrm{SO}(1,1) \times \frac{\mathrm{SO}(5, n-1)}{\mathrm{SO}(5) \times \mathrm{SO}(n-1)}\end{array}$ & $\begin{array}{c}(4, \mathbf{n}) \\
(6, \mathbf{n}-\mathbf{2}) \\
(\mathbf{1}, \mathbf{1})+(5, \mathrm{n}-\mathbf{1})\end{array}$ & $\begin{array}{c}2 \times[(\mathbf{1}, \mathbf{1})+(\mathbf{1}, \mathbf{n})] \\
2 \times[(\mathbf{1}, \mathbf{1})+(\mathbf{6}, \mathbf{1})] \\
2 \times(\mathbf{1}, \mathbf{1})+(\mathbf{5}, \mathbf{1})+ \\
\quad+(\mathbf{1}, \mathbf{n}-\mathbf{1})\end{array}$ \\
\hline 3 & $\frac{\mathrm{SU}(3, n)}{\mathrm{S}[\mathrm{U}(3) \times \mathrm{U}(n)]}$ & $\begin{array}{l}\text { I } \\
\text { II }\end{array}$ & $\begin{array}{c}\mathrm{SU}(2, n) \\
\frac{\mathrm{S}[\mathrm{U}(2) \times \mathrm{U}(n)]}{\mathrm{SU}(3, n-1)} \\
\mathrm{S}[\mathrm{U}(3) \times \mathrm{U}(n-1)] \\
\end{array}$ & $\begin{array}{c}(\mathbf{2}, \mathbf{n})_{n+2}+c . r . \\
(\mathbf{3}, \mathbf{n}-\mathbf{1})_{n+2}+c . r .\end{array}$ & $\begin{array}{l}(\mathbf{1}, \mathbf{n})_{-2}+\text { c.r. } \\
(\mathbf{3}, \mathbf{1})_{1-n}+\text { c.r. }\end{array}$ \\
\hline \multirow{5}{*}{2} & $\begin{array}{c}\frac{\mathrm{SL}(2, \mathbb{R})}{\mathrm{SO}(2)} \times \\
\times \frac{\mathrm{SO}(2, n+2)}{\mathrm{SO}(2) \times \mathrm{SO}(n+2)}\end{array}$ & $\begin{array}{l}\text { I } \\
\text { II } \\
\text { III }\end{array}$ & $\begin{array}{c}- \\
\frac{\mathrm{SO}(2, n)}{\mathrm{SO}(2) \times \mathrm{SO}(n)} \\
\mathrm{SO}(1,1) \times \frac{\mathrm{SO}(1, n+1)}{\mathrm{SO}(n+1)}\end{array}$ & $\begin{array}{c}(\mathbf{2}, \mathbf{n}) \\
\mathbf{1}+(\mathbf{n}+\mathbf{1}) \\
\end{array}$ & $\begin{array}{c}2 \times[(\mathbf{2}, \mathbf{1})+(\mathbf{1}, \mathbf{1})] \\
3 \times \mathbf{1}+(\mathbf{n}+\mathbf{1})\end{array}$ \\
\hline & $\frac{\mathrm{Sp}(6)}{\mathrm{U}(3)}$ & $\begin{array}{l}\text { I } \\
\text { II } \\
\text { III }\end{array}$ & $\begin{array}{c}- \\
\frac{\mathrm{SU}(2,1)}{\mathrm{U}(2)} \\
\frac{\mathrm{SL}(3, \mathbb{R})}{\mathrm{SO}(3)} \\
\end{array}$ & $\begin{array}{c}\mathbf{2}_{-3}+\text { c.r. } \\
\mathbf{5} \\
\end{array}$ & $\begin{array}{c}\mathbf{1}_{-4}+\mathbf{3}_{+2}+c . r . \\
2 \times \mathbf{1}+\mathbf{5}\end{array}$ \\
\hline & $\frac{\mathrm{SU}(3,3)}{\mathrm{S}[\mathrm{U}(3) \times \mathrm{U}(3)]}$ & $\begin{array}{l}\text { I } \\
\text { II } \\
\text { III } \\
\end{array}$ & $\begin{array}{c}- \\
\left(\frac{\mathrm{SU}(2,1)}{\mathrm{U}(2)}\right)^{2} \\
\frac{\mathrm{SL}(3, \mathbb{C})}{\mathrm{SU}(3)} \\
\end{array}$ & $\begin{array}{c}(\mathbf{2}, \mathbf{1})_{3,0}+(\mathbf{1}, \mathbf{2})_{0,3}+\text { c.r. } \\
\mathbf{8}\end{array}$ & $\begin{array}{c}(\mathbf{2}, \mathbf{2})_{1,-1}+(\mathbf{1}, \mathbf{1})_{-2,2}+\text { c.r. } \\
2 \times \mathbf{1}+\mathbf{8} \\
\end{array}$ \\
\hline & $\frac{\mathrm{SO}^{*}(12)}{\mathrm{U}(6)}$ & $\begin{array}{l}\text { I } \\
\text { II } \\
\text { III } \\
\end{array}$ & $\begin{array}{c}- \\
\frac{\mathrm{SU}(4,2)}{\mathrm{S}[\mathrm{U}(4) \times \mathrm{U}(2)]} \\
\frac{\mathrm{SU}^{*}(6)}{\mathrm{USp}(6)} \\
\end{array}$ & $\begin{array}{c}(4,2)_{-3}+\text { c.r. } \\
14 \\
\end{array}$ & $\begin{aligned}(\mathbf{6}, \mathbf{1})_{+2} & +(\mathbf{1}, \mathbf{1})_{-4}+c . r . \\
2 & \times \mathbf{1}+\mathbf{1 4}\end{aligned}$ \\
\hline & $\frac{\mathrm{E}_{7(-25)}}{\mathrm{U}(1) \times \mathrm{E}_{6}}$ & $\begin{array}{c}\text { I } \\
\text { II } \\
\text { III }\end{array}$ & $\begin{array}{c}- \\
\frac{\mathrm{E}_{6(-14)}}{\mathrm{U}(1) \times \mathrm{SO}(10)} \\
\frac{\mathrm{E}_{6(-26)}}{\mathrm{F}_{4}}\end{array}$ & $\begin{array}{c}\overline{\mathbf{1 6}}_{+3}+\text { c.r. } \\
\mathbf{2 6}\end{array}$ & $\begin{array}{c}\mathbf{1}_{+4}+\mathbf{1 0}_{-2}+\text { c.r. } \\
2 \times \mathbf{1}+\mathbf{2 6}\end{array}$ \\
\hline
\end{tabular}

Table 1. Summary of regular, extremal black hole orbits in the various supergravities. The symbols I, II, III denote the $\frac{1}{\mathcal{N}}$-BPS, the non-BPS $\left(I_{4}>0\right)$ and the non-BPS $\left(I_{4}<0\right)$ orbits respectively. For those solutions with non-trivial moduli spaces $\frac{G_{0}}{H_{0}}$ (i.e. $G_{0}$ non-compact), the representations $\mathbf{R}_{0}, \mathbf{R}_{1}$ of $H_{0}$ are given. The symbol "c.r." stands for conjugate representations.

We can write $\mathbb{L}\left(\phi^{r}\right)$ as a $2 n_{V} \times 2 n_{V}$ matrix $\mathbb{L}\left(\phi^{r}\right)_{\hat{N}}^{M}$, where $M$ is an index in the real symplectic representation, while $\hat{N}$ spans a complex basis in which the action of $H$ is blockdiagonal. We can obtain $\mathbb{L}\left(\phi^{r}\right)^{M}{ }_{\hat{N}}$ from the coset representative in the real symplectic 
representation $\mathbb{L}_{S p}\left(\phi^{r}\right)^{M}{ }_{N}$ using the Cayley matrix:

$$
\mathbb{L}\left(\phi^{r}\right)=\mathbb{L}_{S p}\left(\phi^{r}\right) \mathcal{A}^{\dagger} \quad \text { where } \quad \mathcal{A} \equiv \frac{1}{\sqrt{2}}\left(\begin{array}{cc}
\mathbb{1} & \mathrm{i} \mathbb{1} \\
\mathbb{1} & -\mathrm{i} \mathbb{1}
\end{array}\right) .
$$

The central and matter charges $Z_{A B}, Z_{I}$ of the theory can be arranged, together with their complex conjugates, in a $\left(2 n_{V}\right)$-vector $Z_{\hat{M}}$ defined as follows:

$$
Z_{\hat{M}}\left(\phi^{r}, \mathcal{P}\right)=\left(\begin{array}{c}
Z_{A B} \\
Z_{I} \\
\bar{Z}^{A B} \\
\bar{Z}^{I}
\end{array}\right)=-\mathbb{L}\left(\phi^{r}\right)^{T} \mathbb{C} \mathcal{P}=-\mathbb{L}_{1}\left(\phi^{k}\right)^{T} \mathbb{L}_{0}\left(\phi^{\alpha}\right)^{T} \mathbb{C} \mathcal{P},
$$

Now we can use the property of $\mathbb{L}_{0}\left(\phi^{\alpha}\right)$ of being an element of $G_{0}$ in the symplectic representation, so that $\mathbb{L}_{0}^{T} \mathbb{C} \mathcal{P}=\mathbb{C L}_{0}^{-1} \mathcal{P}=\mathbb{C} \mathcal{P}$ and write:

$$
Z_{\hat{M}}\left(\phi^{\alpha}, \phi^{k}, \mathcal{P}\right)=-\mathbb{L}_{1}\left(\phi^{k}\right)^{T} \mathbb{C} \mathcal{P}=Z_{\hat{M}}\left(0, \phi^{k}, \mathcal{P}\right),
$$

that is the central and matter charges do not depend on $\phi^{\alpha}$ at all:

$$
\frac{\partial}{\partial \phi^{\alpha}} Z_{A B}=\frac{\partial}{\partial \phi^{\alpha}} Z_{I}=0 .
$$

Let us now describe the effect of a generic transformation $g_{0}$ in $G_{0}$ on the central charges. From the general properties of coset representatives we know that $D\left(g_{0}\right) \mathbb{L}_{0}\left(\phi^{\alpha}\right)=\mathbb{L}_{0}\left(g_{0}\right.$ * $\left.\phi^{\alpha}\right) D\left(h_{0}\right), D\left(h_{0}\right)$ being a compensator in $H_{0}$ depending on $g_{0}$ and $\phi^{\alpha}$. Now, using the property that $\phi^{k}$ transform in a linear representation of $H_{0}$, we can describe the action of $g_{0}$ on a generic point $\phi$ as follows:

$$
\begin{aligned}
D\left(g_{0}\right) \mathbb{L}\left(\phi^{r}\right) & =D\left(g_{0}\right) \mathbb{L}_{0}\left(\phi^{\alpha}\right) \mathbb{L}_{1}\left(\phi^{k}\right)=\mathbb{L}_{0}\left(g_{0} \star \phi^{\alpha}\right) D\left(h_{0}\right) \mathbb{L}_{1}\left(\phi^{k}\right) D\left(h_{0}\right)^{-1} D\left(h_{0}\right)= \\
& =\mathbb{L}_{0}\left(g_{0} \star \phi^{\alpha}\right) \mathbb{L}_{1}\left(\phi^{\prime k}\right) D\left(h_{0}\right)=\mathbb{L}\left(g_{0} \star \phi^{r}\right) D\left(h_{0}\right),
\end{aligned}
$$

where $\phi^{\prime k}$ is the transformed of $\phi^{k}$ by $h_{0}$, and $\left(g_{0} \star \phi^{\alpha}, \phi^{\prime k}\right)$ define the transformed $g_{0} \star \phi^{r}$ of $\phi^{r}$ by $g_{0}$. From (2.39) and the definition (2.36) we derive the following property:

$$
\forall g_{0} \in G_{0}: Z_{\hat{M}}\left(g_{0} \star \phi^{r}, \mathcal{P}\right)=\left[D\left(h_{0}\right)^{-T}\right]_{\hat{M}}^{\hat{N}} Z_{\hat{N}}\left(\phi^{r}, \mathcal{P}\right)=h_{0} \star Z_{\hat{M}}\left(\phi^{r}, \mathcal{P}\right),
$$

where, to simplify notations we have denoted by $h_{0} \star Z$ the vector $\left[D\left(h_{0}\right)^{-T}\right]_{\hat{M}}{ }^{\hat{N}} Z_{\hat{N}}$. Now consider the $W$ function as a function of $\phi^{r}$ and $\mathcal{P}$ through the central and matter charges $Z_{\hat{M}}$ :

$$
W\left(\phi^{r}, \mathcal{P}\right)=\widehat{W}\left[Z_{\hat{M}}\left(\phi^{r}, \mathcal{P}\right)\right] .
$$

From the duality-invariance of $W$ it follows that, for any $g_{0} \in G_{0}$ we have

$$
W\left(\phi^{r}, \mathcal{P}\right)=W\left(g_{0} \star \phi^{r}, D\left(g_{0}\right) \mathcal{P}\right)=W\left(g_{0} \star \phi^{r}, \mathcal{P}\right) .
$$

Furthermore, using eqs. (2.40), (2.41) we find:

$$
\widehat{W}\left[Z\left(\phi^{r}, \mathcal{P}\right)\right]=W\left(\phi^{r}, \mathcal{P}\right)=W\left(g_{0} \star \phi^{r}, \mathcal{P}\right)=\widehat{W}\left[Z\left(g_{0} \star \phi^{r}, \mathcal{P}\right)\right]=\widehat{W}\left[h_{0} \star Z_{\hat{N}}\left(\phi^{r}, \mathcal{P}\right)\right] .
$$


The above equality holds for any $g_{0} \in G_{0}$ and thus for any $h_{0} \in H_{0}$. We conclude from this that $W$ can be characterized, for a given orbit of solutions, as an $H_{0}$-invariant function of the central and matter charges. This is consistent with what was found in $[3,5]$. Let us stress once more that we have started from a generic charge vector $\mathcal{P}$, so that the definition of $G_{0}$, and thus of $H_{0}$, is charge dependent. We could have started from a given $G_{0}$ inside $G$ and worked out the representative $\mathcal{P}_{0}$ of the $G$-orbit having $G_{0}$ as manifest little group. In this case, by construction, the $\left(\phi^{\alpha}, \phi^{k}\right)$ parametrization is charge-independent.

A detailed analysis. Let us exploit now, for the BPS and non-BPS extremal, regular solutions, the symmetry properties of the $W$ function discussed in the previous sections, to study general aspects of the evolution of the flat and non-flat directions.

We start computing the Killing vectors associated with the $G_{0}$-transformations and write the condition that $W$ be $G_{0}$-invariant in the form of differential equations. To this aim, we will first compute the general expression for the vielbein of $\mathscr{M}_{\text {scal }}$ in the parametrization (2.34). Let us denote by $\left\{T_{\mathcal{A}}\right\}, \mathcal{A}=1, \ldots, \operatorname{dim}\left(G_{0}\right)$, the generators of $G_{0}$. We can perform the Cartan decomposition of the Lie algebras $\mathfrak{g}$ and $\mathfrak{g}_{0}$ generating $G$ and $G_{0}$, respectively, with respect to their maximal compact subalgebras $\mathfrak{h}, \mathfrak{h}_{0}$ :

$$
\mathfrak{g}=\mathfrak{K} \oplus \mathfrak{h}, \quad \mathfrak{g}_{0}=\mathfrak{K}_{0} \oplus \mathfrak{h}_{0} .
$$

Under the adjoint action of $H_{0}$, the space $\mathfrak{K}$ split into subspaces $\mathfrak{K}_{0}$ and $\mathfrak{K}_{1}$ transforming in the representations $\mathbf{R}_{0}, \mathbf{R}_{1}$ of $H_{0}$. The non-compact generators $\left\{\mathbf{K}_{\hat{r}}\right\}, \hat{r}=1 \ldots, n$, of $\mathfrak{K}$ (the indices $\hat{r}, \hat{s}$ label basis elements of the tangent space to the manifold) split into the generators $\left\{\mathbf{K}_{a}\right\}, a, b=1, \ldots, n_{f}$, of $\mathfrak{K}_{0}$, belonging to the tangent space of the submanifold $G_{0} / H_{0}$, and the remaining $n-n_{f}$ generators $\left\{\mathbf{K}_{\hat{k}}\right\}$ of $\mathfrak{K}_{1}$. The Lie algebra $\mathfrak{h}_{0}$ of $H_{0}$ is generated by $\left\{\mathbf{H}_{u}\right\}, u=1 \ldots, \operatorname{dim}\left(H_{0}\right)$. As far as the choice of the parametrization is concerned, for the BPS and non-BPS $\left(I_{4}>0\right)$ solutions, we choose the coset representative as follows:

$$
\mathbb{L}\left(\phi^{r}\right)=\mathbb{L}_{0}\left(\phi^{\alpha}\right) \mathbb{L}_{1}\left(\phi^{k}\right) \in e^{\mathfrak{K}_{0}} \cdot e^{\mathfrak{K}_{1}},
$$

that is $\mathbb{L}_{0}\left(\phi^{\alpha}\right)$ is an element of $e^{\mathfrak{K}_{0}} \equiv G_{0} / H_{0}$ and $\mathbb{L}_{1}\left(\phi^{k}\right)$ is an element of $e^{\mathfrak{K}_{1}}$. This in particular implies that $\phi^{\alpha}$ and $\phi^{k}$ transform in the representations $\mathbf{R}_{0}, \mathbf{R}_{1}$ of $H_{0}$, respectively (see table 1 for a list of these representations).

As for the non-BPS $\left(I_{4}<0\right)$ solutions, it is more convenient to adopt a parametrization of the coset which is different from $(2.45)$, in which $\phi^{k}$ can be defined to transform linearly with respect to the whole $G_{0}$. For this class of solutions, see below, we define $\phi^{k}$ to be parameters of a solvable algebra $\left\{s_{k}\right\}=\left\{s_{\Lambda}, s_{0}\right\}$, generated by $n-n_{f}-1$ nilpotent generators $s_{\Lambda}$ and a Cartan generator $s_{0}$. As we shall see, for the standard choice of the charges $\mathcal{P}_{0}, \phi^{k}$ consist in $n-n_{f}-1$ axions originating from the $D=5$ vector fields and a dilaton describing the modulus of the internal radius in the $D=5 \rightarrow D=4$ dimensional reduction.

We want to compute the components of the vielbein of $\mathscr{M}_{\text {scal }}$ in the basis (2.45). To start with, the $G_{0} / H_{0}$ left-invariant 1 -form reads:

$$
\Omega_{0}\left(\phi^{\alpha}\right)=\mathbb{L}_{0}^{-1} d \mathbb{L}_{0}=\Omega_{0}^{\mathcal{A}} T_{\mathcal{A}}=d \phi^{\beta} \Omega_{0} \mathcal{A}^{\mathcal{A}}\left(\phi^{\alpha}\right) T_{\mathcal{A}} .
$$


If we split the $G_{0}$-generators $T_{\mathcal{A}}$ into generators of $G_{0} / H_{0}\left(\mathbf{K}_{a}\right)$ and of $H_{0}\left(\mathbf{H}_{u}\right)$, that is $\mathcal{A} \rightarrow(\tilde{\alpha}, u)$, then $\Omega_{0}{ }^{b}\left(\phi^{\alpha}\right) \equiv V_{\beta}{ }^{b}\left(\phi^{\alpha}\right)$ defines the vielbein of $G_{0} / H_{0}$. Moreover, let us introduce the left-invariant 1-form:

$$
\Omega_{1}\left(\phi^{k}\right) \equiv \mathbb{L}_{1}^{-1} d \mathbb{L}_{1}=d \phi^{k} V_{k}^{\hat{k}}\left(\phi^{k}\right) \mathbf{K}_{\hat{k}}+\text { connection },
$$

where $d \phi^{k} V_{k}^{\hat{k}}$ will define the vielbein 1-forms along the directions $\mathbf{K}_{\hat{k}}$ of the tangent space. ${ }^{6}$

In terms of the above quantities, we can now compute the left-invariant 1-form of $\mathscr{M}_{\text {scal }}$ in the basis (2.45):

$$
\begin{aligned}
\Omega\left(\phi^{r}\right) & \equiv \mathbb{L}^{-1} d \mathbb{L}=\mathbb{L}_{1}{ }^{-1} \Omega_{0} \mathbb{L}_{1}+\mathbb{L}_{1}^{-1} d \mathbb{L}_{1}=\Omega_{0}^{\mathcal{A}}\left(\phi^{\alpha}\right) \mathbb{L}_{1}^{-1} T_{\mathcal{A}} \mathbb{L}_{1}+\Omega_{1}\left(\phi^{k}\right) \\
& =d \phi^{\beta} \Omega_{0}{ }^{\mathcal{A}}\left(\phi^{\alpha}\right) \mathcal{L}_{1 \mathcal{A}}{ }^{\hat{r}}\left(\phi^{k}\right) \mathbf{K}_{\hat{r}}+d \phi^{k} V_{k}^{\hat{k}} \mathbf{K}_{\hat{k}}+\text { connection },
\end{aligned}
$$

where we have written $\mathbb{L}_{1}{ }^{-1} T_{\mathcal{A}} \mathbb{L}_{1}=\mathcal{L}_{1 \mathcal{A}}{ }^{\hat{r}}\left(\phi^{k}\right) \mathbf{K}_{\hat{r}}+$ compact generators. Similarly we will also write $\mathbb{L}_{0}^{-1} T_{\mathcal{A}} \mathbb{L}_{0}=\mathcal{L}_{0} \mathcal{A}^{\mathcal{B}}\left(\phi^{\alpha}\right) T_{\mathcal{B}}$. The non-vanishing components of the vielbein $\mathcal{V}_{r}{ }^{\hat{s}}$ of $\mathscr{M}_{\text {scal }}$ are now readily computed:

$$
\mathcal{V}_{\beta}{ }^{b}=\Omega_{0} \beta^{\mathcal{A}}\left(\phi^{\alpha}\right) \mathcal{L}_{1 \mathcal{A}}{ }^{b}\left(\phi^{k}\right), \quad \mathcal{V}_{\beta}^{\hat{k}}=\Omega_{0} \beta^{\mathcal{A}}\left(\phi^{\alpha}\right) \mathcal{L}_{1 \mathcal{A}}{ }^{\hat{k}}\left(\phi^{k}\right), \quad \mathcal{V}_{k}{ }^{\hat{k}}=V_{k}{ }^{\hat{k}}\left(\phi^{k}\right) .
$$

Note that for all regular extremal black holes, our choice of parametrization is such that the vielbein matrix has a vanishing off-diagonal block $V_{k}{ }^{a}$, see appendix C.

The non-vanishing blocks of the inverse vielbein $\mathcal{V}^{-1}{ }_{\hat{r}}^{r}$ are:

$$
\mathcal{V}^{-1}{ }_{a}^{\beta}, \quad \mathcal{V}_{\hat{k}}^{-1}, \quad \mathcal{V}^{-1}{ }_{a}^{k}=-\mathcal{V}^{-1}{ }_{a}^{\beta} \mathcal{V}_{\beta}{ }^{\hat{k}} \mathcal{V}_{\hat{k}}^{-1},
$$

where $\mathcal{V}^{-1}{ }_{a}^{\beta}, \mathcal{V}^{-1} \hat{k}^{k}$ are the inverses of the diagonal blocks $\mathcal{V}_{\alpha}{ }^{b}, \mathcal{V}_{k}{ }_{k}$, respectively.

Consider now an infinitesimal $G_{0}$-transformation $g_{0} \sim 1+\epsilon^{\mathcal{A}} T_{\mathcal{A}}, \epsilon^{\mathcal{A}} \sim 0$, and write $\left(g_{0} \star\right.$ $\phi)^{r} \sim \phi^{r}+\epsilon^{\mathcal{A}} k_{\mathcal{A}}^{r}(\phi)$. The Killing vectors $k_{\mathcal{A}}^{r}(\phi)$ are computed, in the parametrization (2.45), to be:

$$
k_{\mathcal{A}}^{r}=\mathcal{L}_{0 \mathcal{A}} \mathcal{B}^{\mathcal{B}}\left(\phi^{\alpha}\right) \mathcal{L}_{1 \mathcal{B}}{ }^{\hat{r}}\left(\phi^{k}\right) \mathcal{V}^{-1}{ }_{\hat{r}}^{r} .
$$

The $G_{0}$-invariance of the $W$-function $\left(W\left(g_{0} \star \phi, \mathcal{P}\right)=W(\phi, \mathcal{P})\right)$ can now be expressed in the following way:

$$
k_{\mathcal{A}}^{r} \frac{\partial W}{\partial \phi^{r}}=0 \Leftrightarrow \mathcal{L}_{1 \mathcal{A}}{ }^{\hat{r}}\left(\phi^{k}\right) \mathcal{V}^{-1}{ }_{r}^{r} \frac{\partial W}{\partial \phi^{r}}=0
$$

where we have used the property that $\mathcal{L}_{0} \mathcal{A}^{\mathcal{B}}(\alpha)$ is non-singular. Using the expression of the vielbein, it will be useful to write the first-order flow-equations for the scalar fields in the following form:

$$
\dot{\phi}^{r} \mathcal{V}_{r}^{\hat{r}}=e^{U} \mathcal{V}^{-1 \hat{r} s} \frac{\partial W}{\partial \phi^{s}} \Leftrightarrow\left\{\begin{array}{l}
\dot{\phi}^{\beta} \mathcal{V}_{\beta}{ }^{a}=e^{U} \mathcal{V}^{-1 a r} \frac{\partial W}{\partial \phi^{r}} \\
\dot{\phi}^{r} \mathcal{V}_{r}{ }^{\hat{k}}=e^{U} \mathcal{V}^{-1 \hat{k} k} \frac{\partial W}{\partial \phi^{k}}
\end{array}\right.
$$

We shall illustrate the implications of the above formula in two relevant cases: The BPS solution and the non-BPS one with $I_{4}<0$.

\footnotetext{
${ }^{6}$ The reason why the left-invariant 1 -form $\Omega_{1}$ in eq. (2.47) does not expand on the generators $\mathbf{K}_{a}$ will be clarified in appendix C.
} 
The BPS black holes. For the sake of concreteness we shall consider the supersymmetric regular solutions $\left(\frac{1}{8}\right.$-BPS $)$ in the maximal theory $\mathcal{N}=8$, although our discussion is easily extended to non-maximal theories. In this case $G_{0}=\mathrm{E}_{6(+2)}$ and $H_{0}=\mathrm{SU}(2) \times \mathrm{SU}(6) \subset$ $\mathrm{SU}(8)=\mathrm{H}$. With respect to the adjoint action of $H_{0}$, the coset space $\mathfrak{K}$, in the $\mathbf{7 0}$ of SU(8), splits into the subspaces $\mathfrak{K}_{0}=\left\{\mathbf{K}_{a}\right\}$ in the $(\mathbf{2}, \mathbf{2 0})$ and $\mathfrak{K}_{1}=\left\{\mathbf{K}_{\hat{k}}\right\}$ in the $(\mathbf{1}, \mathbf{1 5}) \oplus(\mathbf{1}, \overline{\mathbf{1 5}})$ of $H_{0}$, according to the branching:

$$
70 \rightarrow(2,20) \oplus(\mathbf{1}, 15) \oplus(\mathbf{1}, \overline{15}) .
$$

The parametrization (2.34) amounts to the following choice of the coset representative:

$$
\mathbb{L}=\mathbb{L}_{0}\left(\phi^{\alpha}\right) \mathbb{L}_{1}\left(\phi^{k}\right), \quad \mathbb{L}_{0}\left(\phi^{\alpha}\right) \in e^{\mathfrak{K}_{0}}, \quad \mathbb{L}_{1}\left(\phi^{k}\right) \in e^{\mathfrak{K}_{1}} .
$$

Since the index $a$ spans a $\mathrm{SU}(2)$-doublet $(a=(A, \lambda), A=1,2, \lambda=[m n p]=1, \ldots, 20$, $m, n, p=1, \ldots, 6)$, while $\hat{k}$ only $\mathrm{SU}(2)$-singlets, being $\phi^{k}$ themselves $\mathrm{SU}(2)$-singlets, the non vanishing components of the matrix $\mathcal{L}_{1 \mathcal{A}}{ }^{\hat{r}}$ are: $\left.\mathcal{L}_{1 a^{b}}{ }^{b} \phi^{k}\right), \mathcal{L}_{1 u}{ }^{\hat{k}}\left(\phi^{k}\right)$. Consider now the implications of the $G_{0}$-invariance of $W$, as expressed by eq. (2.52). The $H_{0}=\mathrm{SU}(2) \times \mathrm{SU}(6)$ invariance corresponds to the $\mathcal{A}=u$ component of the equation, and implies

$$
\mathcal{L}_{1 u}^{\hat{k}}\left(\phi^{k}\right) \mathcal{V}_{\hat{k}}^{-1}\left(\phi^{k}\right) \frac{\partial W}{\partial \phi^{k}}=0
$$

The invariance of $W$ under $G_{0} / H_{0}$-transformations, on the other hand, implies, using (2.50) and (2.49):

$$
\begin{aligned}
0 & =\mathcal{L}_{1 a}{ }^{b}\left(\phi^{k}\right) \mathcal{V}^{-1}{ }_{b}{ }^{r} \frac{\partial W}{\partial \phi^{r}}=\mathcal{L}_{1 a}{ }^{b}\left(\phi^{k}\right)\left[\mathcal{V}^{-1}{ }_{b}{ }^{\gamma} \frac{\partial W}{\partial \phi^{\gamma}}+\mathcal{V}^{-1}{ }_{b}{ }^{k} \frac{\partial W}{\partial \phi^{k}}\right]= \\
& =\mathcal{L}_{1 a}{ }^{b}\left(\phi^{k}\right)\left[\mathcal{V}^{-1}{ }_{b}{ }^{\gamma} \frac{\partial W}{\partial \phi^{\gamma}}-\mathcal{V}^{-1}{ }_{b}{ }^{\gamma} \Omega_{0 \gamma}{ }^{u} \mathcal{L}_{1 u}{ }^{\hat{k}} \mathcal{V}^{-1}{ }_{\hat{k}}{ }^{k} \frac{\partial W}{\partial \phi^{k}}\right]= \\
& =\mathcal{L}_{1 a}{ }^{b}\left(\phi^{k}\right) \mathcal{V}^{-1}{ }_{b}{ }^{\gamma} \frac{\partial W}{\partial \phi^{\gamma}} \Rightarrow \frac{\partial W}{\partial \phi^{\alpha}}=0,
\end{aligned}
$$

where we have used eq. (2.56) and the property that the block $\mathcal{L}_{1 a}{ }^{b}\left(\phi^{k}\right)$ is non-singular. The above equation expresses the $\phi^{\alpha}$-independence of $W$, which we had proven before in a different way. Finally, consider the evolution of the $\phi^{\alpha}$-scalars as described in (2.53). From equation (2.57) it follows that:

$$
\dot{\phi}^{\beta} \mathcal{V}_{\beta}^{a}=e^{U} \mathcal{V}^{-1 a r} \frac{\partial W}{\partial \phi^{r}}=0
$$

namely the flat directions $\phi^{\alpha}$ are constant along the flow. This is consistent with the $\mathcal{N}=2$ supersymmetry of the solution, since the variation of the fermions $\lambda^{m n p}$ (the hyperinos in the $\mathcal{N}=2$ truncation, in the $\mathbf{2 0}$ of $\mathrm{SU}(6))$ on the solution reads:

$$
\delta \lambda^{m n p} \propto \dot{\phi}^{\alpha} \mathcal{V}_{\alpha}^{A, m n p} \epsilon_{A}=0
$$

where, as usual, we have written $a=(A, m n p)$.

As far as the non-BPS black holes with $I_{4}>0$ are concerned, the analysis is analogous to the BPS case illustrated above. 
Non-BPS black holes with $\boldsymbol{I}_{\mathbf{4}}<\mathbf{0}$. In this case the little group $G_{0}$ of the charge vector is the duality group of the five-dimensional parent theory (for the $\mathcal{N}=8$ case $G_{0}=\mathrm{E}_{6(6)}$ ), so that the flat directions $\left(\phi^{\alpha}\right)$ spanning $G_{0} / H_{0}$ are the five-dimensional scalar fields. We can use the solvable parametrization for $\mathscr{M}_{\text {scal }}$ by writing $\mathscr{M}_{\text {scal }}=\exp (S o l v)$, where $S o l v$ is the solvable Lie algebra defined by the Iwasawa decomposition of $G$ with respect to $H$. Let moreover $S_{\text {olv }}$ be the solvable Lie algebra generating the submanifold spanned by the flat directions: $G_{0} / H_{0} \equiv \exp \left(\operatorname{Solv}_{0}\right)$.

In the solvable parametrization the moduli $\phi^{\alpha}$ are parameters of the generators $s_{\alpha}$ of Solv ${ }^{7} \cdot$ We can decompose the scalars $\phi^{r}$ into $\phi^{\alpha}$ and $\phi^{k}$ by decomposing $S o l v$ with respect to $\operatorname{Solv}_{0}$ :

$$
\text { Solv }=\mathfrak{o}(1,1) \oplus \text { Solv }_{0} \oplus \mathbf{R}_{-2},
$$

where the $\mathfrak{o}(1,1)$ generator $s_{0}$ is parametrized by the modulus $\sigma_{0}$ of the radius of the fifth dimension and the abelian subalgebra $\mathbf{R}_{-2}=\left\{s_{\Lambda}\right\}$ is parametrized by the axions $\sigma^{\Lambda}$ originating from the five-dimensional vector fields and transforming according to the representation $\overline{\mathbf{R}}$ of $G_{0}$ with $\mathrm{O}(1,1)$-grading +2 (in the maximal theory $\mathbf{R}=\mathbf{2 7}$ ). The decomposition (2.60) originates from the general branching rule of $G$ with respect to $G_{0}$

$$
\operatorname{Adj}(G)=\mathbf{1}_{0} \oplus \operatorname{Adj}(G) \oplus \mathbf{R}_{-2} \oplus \overline{\mathbf{R}}_{+2},
$$

The non-flat directions $\phi^{k}$ therefore consist of $\sigma_{0}$ and $\sigma^{\Lambda}$, which transform in a representation of $G_{0}$. The following commutation relations hold:

$$
\left[T_{\mathcal{A}}, s_{0}\right]=0, \quad\left[s_{0}, s_{\Lambda}\right]=+2 s_{\Lambda}, \quad\left[T_{\mathcal{A}}, s_{\Lambda}\right]=-T_{\mathcal{A} \Lambda}{ }^{\Sigma} s_{\Sigma} .
$$

We shall write $\mathbb{L}_{1}\left(\phi^{k}\right)=\mathbb{L}\left(\sigma^{\Lambda}\right) e^{\sigma_{0} s_{0}}$.

Note that the coset parametrizations that we are using throughout this section, defined in eq. (2.45), differ from the standard parametrization of $\mathscr{M}_{\text {scal }}$, which originates from the $D=5 \rightarrow D=4$ reduction (like, for instance, the special coordinate parametrization of the special Kähler manifold in the $\mathcal{N}=2$ theory). The standard parametrization corresponds indeed to the following choice of the coset representative:

$$
\mathbb{L}\left(\phi^{r}\right)=\mathbb{L}\left(\tilde{\sigma}^{\Lambda}\right) e^{\sigma_{0} s_{0}} \mathbb{L}_{0}\left(\phi^{\alpha}\right) .
$$

The prescription (2.34), that we are using here, yields instead a different parametrization in which the order of the factors in the coset representative is different: $\mathbb{L}\left(\phi^{r}\right)=$ $\mathbb{L}_{0}\left(\phi^{\alpha}\right) \mathbb{L}\left(\sigma^{\Lambda}\right) e^{\sigma_{0} s_{0}}$. The two parametrizations are related by a redefinition of the axions:

$$
\tilde{\sigma}^{\Lambda}=\mathbb{L}_{0}^{-1} \Sigma^{\Lambda}\left(\phi^{\alpha}\right) \sigma^{\Sigma},
$$

where $\mathbb{L}_{0 \Sigma^{\Lambda}}\left(\phi^{\alpha}\right)$ is the matrix form of $\mathbb{L}_{0}\left(\phi^{\alpha}\right)$ in the $\mathbf{R}$ representation: $\mathbb{L}_{0}\left(\phi^{\alpha}\right)^{-1} s_{\Sigma} \mathbb{L}_{0}\left(\phi^{\alpha}\right)=\mathbb{L}_{0} \Sigma^{\Lambda}\left(\phi^{\alpha}\right) s_{\Lambda}$. The vielbein 1-forms $d \phi^{r} \mathcal{V}_{r}^{\hat{r}}$ are defined, as usual, as the components of the left-invariant 1-form along the non compact generators

\footnotetext{
${ }^{7}$ Note that, in contrast to the parametrization used for the other classes of black holes, neither $\phi^{\alpha}$ nor the corresponding solvable generators $s_{\alpha}$ transform, under the adjoint action of $H_{0}$, in a linear representation.
} 
$\mathbf{K}_{\hat{k}} \propto\left(s_{r}+s_{r}^{\dagger}\right)$. The non-vanishing components of the vielbein matrix $\mathcal{V}_{r}{ }^{\hat{r}}$ and of its inverse $\mathcal{V}^{-1}{ }_{\hat{r}} r$ are readily computed to be:

$$
\begin{aligned}
& \mathcal{V}_{\alpha}{ }^{b}\left(\phi^{\alpha}\right), \quad \mathcal{V}_{\alpha}^{\hat{\Lambda}}=-e^{-2 \sigma_{0}} V_{\alpha}{ }^{b}\left(\phi^{\alpha}\right) s_{b \Sigma}{ }^{\hat{\Lambda}} \sigma^{\Sigma}, \quad \mathcal{V}_{\Lambda}{ }^{\hat{\Sigma}}=e^{-2 \sigma_{0}} \delta_{\Lambda}{ }^{\hat{\Sigma}}, \quad \mathcal{V}_{0}{ }^{\hat{0}}=1, \\
& \mathcal{V}^{-1}{ }_{a}{ }^{\beta}\left(\phi^{\alpha}\right), \quad \mathcal{V}^{-1}{ }_{a}^{\Lambda}=s_{a}{ }^{\Lambda} \sigma^{\Sigma}, \mathcal{V}^{-1}{ }_{\hat{\Lambda}}{ }^{\Sigma}=e^{2 \sigma_{0}} \delta_{\hat{\Lambda}}{ }^{\Sigma}, \quad \mathcal{V}^{-1}{ }_{\hat{0}}{ }^{0}=1,
\end{aligned}
$$

where $s_{a \Sigma} \Sigma^{\Lambda}$ is the matrix form of the generator $s_{a}$ of $S o l v_{0}$ in the representation $\mathbf{R}$. Consider now the $G_{0}$-invariance condition on $W$, as expressed by eq. (2.52) and use the following property:

$$
\mathbb{L}_{1}{ }^{-1} T_{\mathcal{A}} \mathbb{L}_{1}=T_{\mathcal{A}}-e^{-2 \sigma_{0}} T_{\mathcal{A} \Sigma}{ }^{\Lambda} \sigma^{\Sigma}=\mathcal{L}_{\mathcal{A}}{ }^{\hat{r}} \mathbf{K}_{\hat{r}}+\text { compact generators . }
$$

After some algebra we find that the $H_{0}$-invariance of $W$ (component $\mathcal{A}=u$ of eq. (2.52)) implies:

$$
H_{u \Sigma} \Lambda^{\Sigma} \sigma^{\Sigma} \frac{\partial W}{\partial \sigma^{\Lambda}}=0
$$

while the invariance with respect to $G_{0} / H_{0}$ (component $\mathcal{A}=a$ of the same equation) implies:

$$
\frac{\partial W}{\partial \phi^{\alpha}}=0
$$

that is $W$ must be $\alpha$-independent, as expected by other arguments. Let us note however that now the $\phi^{\alpha}$ are evolving since:

$$
\dot{\phi}^{a} \mathcal{V}_{\alpha a}=e^{U} s_{a \Sigma}{ }^{\Lambda} \sigma^{\Sigma} \frac{\partial W}{\partial \sigma^{\Lambda}} \neq 0
$$

since the right hand side represents the variation of $W$ corresponding to an infinitesimal $G_{0} / H_{0}$ transformation of $\sigma^{\Lambda}$ and $W$ is invariant only with respect to $H_{0}$-transformations of $\sigma^{\Lambda}$ (see equation (2.67)). One can easily verify that the flow of the non-flat scalars $\left(\sigma_{0}, \sigma^{\Lambda}\right.$ ) is described by an $\alpha$-independent dynamical system which has an equilibrium point for $\frac{\partial W}{\partial \sigma^{\Lambda}}=\frac{\partial W}{\partial \sigma_{0}}=0$, at which, by virtue of (2.69), also $\dot{\alpha}=0$. Indeed, using eqs. (2.53) and the explicit form of the vielbein matrix and of its inverse (2.65), we can substitute in the equations for $\phi^{k}$ the expression of $\dot{\alpha}^{a}$ and find for the non-flat directions the following equations:

$$
\dot{\sigma}^{\Lambda}=e^{U}\left(e^{4 \sigma_{0}} \delta^{\Lambda \Sigma}+s^{a}{ }^{\Lambda}{ }^{\Lambda} s_{a \Gamma^{\Sigma}} \sigma^{\Delta} \sigma^{\Gamma}\right) \frac{\partial W}{\partial \sigma^{\Sigma}}, \quad \dot{\sigma}_{0}=e^{U} \frac{\partial W}{\partial \sigma_{0}} .
$$

According to the above equations, the non-flat directions $\sigma^{\Lambda}, \sigma_{0}$ evolve towards fixed values at the horizon which depend only on the quantized charges and solve the equilibrium conditions $\frac{\partial W}{\partial \sigma^{\Lambda}}=\frac{\partial W}{\partial \sigma_{0}}=0$. Only the flat directions can depend at the horizon on the values of the scalar fields at radial infinity, but this is not in contradiction with the attractor mechanism since the near horizon geometry only depends on the corresponding values of $\sigma^{\Lambda}, \sigma_{0}$, through $V$ or $W$.

Let us finally give an example of the $\left(\phi^{\alpha}, \phi^{k}\right)$-parametrization in the STU model, in the case $I_{4}(\mathcal{P})<0$, and show that the central and matter charges do not depend on $\alpha$. 
The STU model is a $\mathcal{N}=2$ supergravity with $n=6$ real scalar fields (i.e. 3 complex ones $\left.\{s, t, u\} \equiv\left\{z_{1}, z_{2}, z_{3}\right\}\right)$ belonging to three vector multiplets. The number of vector fields is $n_{V}=4$. The scalar manifold has the following form:

$$
\mathscr{M}_{S T U}=\left(\frac{\mathrm{SL}(2, \mathbb{R})}{\mathrm{SO}(2)}\right)_{s} \times\left(\frac{\mathrm{SL}(2, \mathbb{R})}{\mathrm{SO}(2)}\right)_{t} \times\left(\frac{\mathrm{SL}(2, \mathbb{R})}{\mathrm{SO}(2)}\right)_{u}
$$

where each factor is parametrized by the complex scalars $s=a_{1}^{\prime}-\mathrm{i} e^{\varphi_{1}}, t=a_{2}^{\prime}-\mathrm{i} e^{\varphi_{2}}, u=$ $a_{3}^{\prime}-\mathrm{i} e^{\varphi_{3}}$. The eight quantized charges transform in the $(\mathbf{2}, \mathbf{2}, \mathbf{2})$ of the isometry group $G=\mathrm{SL}(2, \mathbb{R})^{3}$ and in this representation the coset representative is the tensor product of the coset representatives of each factor in (2.71) in the fundamental representation of $\operatorname{SL}(2, \mathbb{R})$ :

$$
\mathbb{L}\left(z_{i}\right)=\mathbb{L}_{1}\left(z_{1}\right) \otimes \mathbb{L}_{2}\left(z_{2}\right) \otimes \mathbb{L}_{3}\left(z_{3}\right)
$$

where each $2 \times 2$ matrix has the following form:

$$
\mathbb{L}_{i}\left(z_{i}\right)=\left(\begin{array}{cc}
1 & 0 \\
-a_{i}^{\prime} & 1
\end{array}\right)\left(\begin{array}{cc}
e^{-\frac{\varphi_{i}}{2}} & 0 \\
0 & e^{\frac{\varphi_{i}}{2}}
\end{array}\right) .
$$

In this case the $\sigma^{\prime \Lambda}$ axions are nothing but $a_{1}^{\prime}, a_{2}^{\prime}, a_{3}^{\prime}$. The little group of the $I_{4}(\mathcal{P})<0$ orbit is $G_{0}=\mathrm{O}(1,1)^{2}$. For generic charges, like for instance those corresponding to the $\overline{D 0}, D 4$ system $\left(q_{0}, p^{i}\right)$, the action of $G_{0}$ is rather involved and depends on the charges themselves. We can consider however, as representative of the same $G$-orbit, the charges corresponding to the $D 0-D 6$ system $\left(p^{0}, q_{0}\right)$. In this case $G_{0}$ is parametrized by two combinations of the dilatons $\varphi_{i}:\left\{\phi^{\alpha}\right\}_{\alpha=1,2}=\left\{\phi^{1}=\frac{1}{\sqrt{2}}\left(\varphi_{1}-\varphi_{2}\right), \phi^{2}=\frac{1}{\sqrt{6}}\left(\varphi_{1}+\varphi_{2}-2 \varphi_{3}\right)\right\}$. According to the general prescription (2.34), the part $\mathbb{L}_{0}$ of the coset representative depending on the flat directions $\phi^{1}, \phi^{2}$, should be the left factor of the product. This corresponds to bringing the diagonal dilatonic factor in (2.73) to the left and redefining the axion:

$$
\mathbb{L}_{i}\left(z_{i}\right)=\left(\begin{array}{cc}
e^{-\frac{\varphi_{i}}{2}} & 0 \\
0 & e^{\frac{\varphi_{i}}{2}}
\end{array}\right)\left(\begin{array}{cc}
1 & 0 \\
-a_{i} & 1
\end{array}\right),
$$

where $a_{i}=a_{i}^{\prime} e^{-\varphi_{i}}$. The three complex scalar fields, in this new parametrization, read: $z_{i}=e^{\varphi_{i}}\left(a_{i}-\mathrm{i}\right)$. The central and matter charges have the following form:

$$
\begin{aligned}
Z & =\frac{e^{\frac{\sqrt{3}}{2} \sigma_{0}}}{2 \sqrt{2}}\left[q_{0}+p^{0} e^{\sqrt{3} \sigma_{0}}\left(a_{1}-\mathrm{i}\right)\left(a_{2}-\mathrm{i}\right)\left(a_{3}-\mathrm{i}\right)\right], \\
Z_{1} & =\frac{e^{\frac{\sqrt{3}}{2} \sigma_{0}}}{2 \sqrt{2}}\left[q_{0}+p^{0} e^{\sqrt{3} \sigma_{0}}\left(a_{1}+\mathrm{i}\right)\left(a_{2}-\mathrm{i}\right)\left(a_{3}-\mathrm{i}\right)\right], \\
Z_{2} & =\frac{e^{\frac{\sqrt{3}}{2} \sigma_{0}}}{2 \sqrt{2}}\left[q_{0}+p^{0} e^{\sqrt{3} \sigma_{0}}\left(a_{1}-\mathrm{i}\right)\left(a_{2}+\mathrm{i}\right)\left(a_{3}-\mathrm{i}\right)\right], \\
Z_{3} & =\frac{e^{\frac{\sqrt{3}}{2} \sigma_{0}}}{2 \sqrt{2}}\left[q_{0}+p^{0} e^{\sqrt{3} \sigma_{0}}\left(a_{1}-\mathrm{i}\right)\left(a_{2}-\mathrm{i}\right)\left(a_{3}+\mathrm{i}\right)\right],
\end{aligned}
$$


where $\sigma_{0} \equiv \frac{1}{\sqrt{3}}\left(\varphi_{1}+\varphi_{2}+\varphi_{3}\right)$. We observe that none of the central and matter charges depend on the scalars $\left\{\phi^{\alpha}\right\}=\left\{\phi^{1}, \phi^{2}\right\}$, but only on the remaining scalar fields $\left\{\phi^{k}\right\}$, $k=3, \ldots, 6$, defined as follows:

$$
\left\{\phi^{k}\right\}=\left\{\sigma_{0}, a_{i} \equiv a_{i}^{\prime} e^{-\varphi_{i}}\right\} .
$$

The scalars $\phi^{\alpha}$ are then flat directions of any function of the central and matter charges, including $V$ and $W$.

\section{Small black holes in the $\mathcal{N}=8$ theory}

$\mathcal{N}=8$ supergravity admits two orbits for "large" extremal black holes (one of which is $1 / 4$-BPS and the other a non-BPS one) and three orbits for "small" extremal black holes (all of them BPS, preserving $1 / 8,1 / 4$, and $1 / 2$ supersymmetry respectively).

Following the analysis of [24], the ADM mass for the three small orbits is given by the largest eigenvalue of the central charge matrix $Z_{A B}$. Its eigenvalues for $1 / 8$ and $1 / 4$ BPS solutions are given by the quartic and quadratic roots of the secular equation

$$
\prod_{i=1}^{4}\left(\lambda-\lambda_{i}\right)=0 \quad\left(\lambda_{i}=\rho_{i}^{2}\right)
$$

$\rho_{i}$ being the skew-eigenvalue of $Z_{A B}$ when written in normal form. In particular we have:

- For $1 / 8$ BPS: $\lambda_{1}>\lambda_{2} \geq \lambda_{3} \geq \lambda_{4}$

- For 1/4 BPS: $\lambda_{1}=\lambda_{2}>\lambda_{3}=\lambda_{4}$

- For $1 / 2$ BPS: $\lambda_{i}=\lambda \forall i=1, \cdots, 4$.

The five $\mathcal{N}=8$ orbits preserve, respectively, the following symmetries:

$$
\begin{aligned}
& \text { - large: }\left\{\begin{array}{cc}
1 / 8 \text { BPS: } & \mathrm{SU}(2) \times \mathrm{SU}(6) \\
\text { non-BPS: } & \mathrm{USp}(8)
\end{array}\right. \\
& \text { - small }\left\{\begin{array}{cc}
1 / 8 \text { BPS: } & \mathrm{USp}(2) \times \mathrm{USp}(6) \\
1 / 4 \text { BPS: } & \mathrm{SU}(4) \times \mathrm{USp}(4) \\
1 / 2 \text { BPS: } & \mathrm{USp}(8)
\end{array}\right.
\end{aligned}
$$

The superpotential $W$, for all the BPS orbits, is given by the highest eigenvalue of the central charge matrix $Z_{A B}$. However one can also get small orbits from the large non-BPS orbit, in the limiting procedure $I_{4} \rightarrow 0$. Indeed, in this limit the non-BPS orbit becomes supersymmetric, the fraction of supersymmetry preserved depending on whether further constraints on $I_{4}$ are imposed. For example, let us start with a non-BPS black hole with charges $\left(p^{0}, q_{0}\right)$ turned on. It has $I_{4}=-\left(p_{0} q^{0}\right)^{2}$ and $\operatorname{symmetry} \operatorname{USp}(8)$. The limit $I_{4}=0$, obtained for $p_{0} q^{0}=0$, gives a $1 / 2$ BPS black home which has the same USp(8) symmetry. For the most general $W$ of a non-BPS configuration, as defined in [5], the $I_{4}=0$ limit just gives back eq. (3.1) with $\lambda=W^{2}$. 


\section{Small black holes in the $\mathcal{N}=4$ theory}

The moduli space of $\mathcal{N}=4$ supergravity is

$$
\mathscr{M}_{\text {scal }}=\frac{\mathrm{SO}(1,2)}{\mathrm{SO}(2)} \times \frac{\mathrm{SO}(6, \mathrm{n})}{\mathrm{SO}(6) \times \mathrm{SO}(\mathrm{n})} .
$$

The presence of a non-simple U-duality group $G=\mathrm{SO}(1,2) \times \mathrm{SO}(6, \mathrm{n})$ makes the analysis more involved here than in the $\mathcal{N}=8$ case, and requires to explicitly set the notations.

We consider static and spherically symmetric extremal black holes. In particular we address our study to the small orbits of the theory, corresponding to a vanishing value of the horizon area. They are identified by a vanishing quartic invariant of the U-duality group.

The electric and magnetic charges span the representation $(\mathbf{2}, \mathbf{6}+\mathbf{n})$ of the U-duality group $\mathrm{SU}(1,1) \times \mathrm{SO}(6, \mathrm{n}):^{8}$

$$
\mathcal{P}_{\Lambda}^{a}, \quad a=1,2, \quad \Lambda=1, \cdots, 6+n,
$$

such that

$$
\mathcal{P}_{\Lambda}^{1}=p_{\Lambda}=\eta_{\Lambda \Sigma} p^{\Sigma}, \quad \mathcal{P}_{\Lambda}^{2}=q_{\Lambda} .
$$

Here $\eta_{\Lambda \Sigma}=\operatorname{diag}(\overbrace{+, \cdots,+}^{6}, \overbrace{-, \cdots,-}^{n})$ is the $\operatorname{SO}(6, n)$-invariant metric.

It is useful to introduce an $\mathrm{SO}(1,2)$-invariant tensor quadratic in the charges:

$$
T_{\Lambda \Sigma} \equiv \frac{1}{2}\left(p_{\Lambda} q_{\Sigma}-q_{\Lambda} p_{\Sigma}\right)
$$

and an $\mathrm{SO}(6, \mathrm{n})$-invariant tensor, quadratic in the charges, in the adjoint representation of $\mathrm{SO}(1,2)$, obtained as follows:

$$
L^{a}=\frac{1}{2} \gamma_{\alpha \beta}^{a} \mathcal{P}^{\alpha} \cdot \mathcal{P}^{\beta}
$$

where $\gamma_{\alpha \beta}^{a}=\left(\mathbb{1},-\sigma_{3}, \sigma_{1}\right)_{\alpha \beta}$ and $\sigma^{i}$ denote the Pauli matrices. The indices $a$ in the $\mathbf{3}$ of $\mathrm{SO}(1,2)$ are lowered and raised with the metric $\eta_{a b}=\operatorname{diag}(+,-,-)$. The explicit form of the 3 -dimensional vector $L^{a}$ is given by:

$$
L^{0}=\frac{1}{2}\left(p^{2}+q^{2}\right), \quad L^{1}=\frac{1}{2}\left(p^{2}-q^{2}\right), \quad L^{2}=p \cdot q
$$

where $p^{2} \equiv p^{\Lambda} p_{\Lambda}, q^{2} \equiv q^{\Lambda} q_{\Lambda}, p \cdot q \equiv p^{\Lambda} q_{\Lambda}$.

The quartic U-invariant of the theory is given by

$$
I_{4}=\epsilon_{\alpha \beta} \mathcal{P}_{\Lambda}^{\alpha} \mathcal{P}_{\Sigma}^{\beta} \epsilon_{\gamma \delta} \mathcal{P}^{\gamma \Lambda} \mathcal{P}^{\delta \Sigma}=p^{2} q^{2}-(p \cdot q)^{2}=L^{a} L^{b} \eta_{a b}=2 T_{\Lambda \Sigma} T^{\Lambda \Sigma}
$$

The covariant tensors $T_{\Lambda \Sigma}$ and $L^{a}$ can be expressed in terms of derivatives of the quartic invariant, restricted to the adjoint representation of the two subgroups of the U-duality

\footnotetext{
${ }^{8}$ In this and in the following sections the indices $r, a, \alpha, \Lambda$ have different range and definition with respect to the previous sections. Their definition will be given as soon as they are introduced.
} 
group as [24]:

$$
\begin{aligned}
T_{\Lambda \Sigma} & =\frac{1}{24} \epsilon^{\alpha \beta} \frac{\partial^{2} I_{4}}{\partial \mathcal{P}_{\alpha}^{\Lambda} \partial \mathcal{P}_{\beta}^{\Sigma}}=\left.\frac{\partial^{2} I_{4}}{\partial \mathcal{P}^{2}}\right|_{(\mathbf{1}, \operatorname{Adj}(\operatorname{SO}(6, \mathrm{n})))} \\
L^{a} & =\frac{1}{8(5+n)} \gamma^{i \mid \alpha \beta} \frac{\partial^{2} I_{4}}{\partial \mathcal{P}_{\alpha}^{\Lambda} \partial \mathcal{P}_{\beta \Lambda}}=\left.\frac{\partial^{2} I_{4}}{\partial \mathcal{P}^{2}}\right|_{(\operatorname{Adj}(\operatorname{SO}(1,2)), \mathbf{1})}
\end{aligned}
$$

For the subsequent analysis we shall use the expression of $I_{4}$ in terms of the central and matter charges given in (2.15). This formula can be understood by noting that the vector

$$
\tilde{L}^{a}=\left(S_{1}, \operatorname{Re}\left(S_{2}\right), \operatorname{Im}\left(S_{2}\right)\right)
$$

transforms in the 3 of $\mathrm{SO}(1,2)$, being related to $L^{a}$ through the action of the coset representative of the $\frac{\mathrm{SO}(1,2)}{\mathrm{SO}(2)}$ factor in the same representation. Therefore the 2 vectors $\tilde{L}^{a}$ and $L^{a}$ are in the same duality orbit (indeed they coincide in the origin of the $\frac{\mathrm{SO}(1,2)}{\mathrm{SO}(2)}$ factor). This is a relation between dressed and bare charges which will be very useful in the sequel.

When $I_{4} \geq 0$, the sign of $L^{0}$ (and hence of $S_{1}$ ) has a U-duality invariant meaning. Indeed, $I_{4}$ represents the norm of the vector $L^{a}$. Using the terminology of the Lorentz group, a positive or null norm vector $L^{a}$, being "time-like" or "light-like" respectively, has the sign of its time component invariant under $\mathrm{SO}(1,2)$ transformations. Viceversa, if $I_{4}<0 L^{a}$ is space-like and the sign of its time-component has no invariant meaning.

Exploiting the symmetries of the theory, the central charge matrix can always be reduced to the normal form $Z_{A B} \rightarrow\left(\begin{array}{cc}z_{1} \epsilon & 0 \\ 0 & z_{2} \epsilon\end{array}\right)$ with skew eigenvalues $z_{1}, z_{2} \in \mathbb{R}$, while the matter charge-vector $Z_{I}=\rho_{I} e^{\mathrm{i} \theta_{I}}$ can always be reduced to a form where the first two components are $\rho_{1} e^{\mathrm{i} \theta}$ and $\rho_{2}$, all the other entries being zero [2, 24]. In this normal frame $S_{1}$ and $S_{2}$ take the simple form:

$$
\begin{aligned}
& S_{1}=z_{1}^{2}+z_{2}^{2}-\rho_{1}^{2}-\rho_{2}^{2} \\
& S_{2}=2 z_{1} z_{2}-\rho_{1}^{2} e^{2 \mathrm{i} \theta}-\rho_{2}^{2},
\end{aligned}
$$

and the general expression for the quartic invariant in terms of dressed charges in the normal frame reads:

$$
I_{4}=\left(z_{1}-z_{2}\right)^{2}\left[\left(z_{1}+z_{2}\right)^{2}-2\left(\rho_{1}^{2}+\rho_{2}^{2}\right)\right]+2 \rho_{1}^{2}\left(\rho_{2}^{2}-2 z_{1} z_{2}\right)(1-\cos (2 \theta)) .
$$

We shall however use a different normal form for the matter charges, in which central and matter charges appear in a more symmetric fashion.

$$
\left(\begin{array}{c}
Z_{1}^{\prime} \\
Z_{2}^{\prime}
\end{array}\right) \equiv \frac{1}{\sqrt{2}}\left(\begin{array}{cc}
1 & \mathrm{i} \\
1 & -\mathrm{i}
\end{array}\right)\left(\begin{array}{c}
Z_{1} \\
Z_{2}
\end{array}\right)=\frac{1}{\sqrt{2}}\left(\begin{array}{c}
\rho_{1} e^{\mathrm{i} \theta}+\mathrm{i} \rho_{2} \\
\rho_{1} e^{\mathrm{i} \theta}-\mathrm{i} \rho_{2}
\end{array}\right)=e^{\mathrm{i} \varphi}\left(\begin{array}{c}
\widetilde{\rho}_{1} e^{\mathrm{i} \beta} \\
\widetilde{\rho}_{2} e^{-\mathrm{i} \beta}
\end{array}\right) .
$$

The phase $\beta$ can be absorbed by a $\mathrm{SO}(2) \subset \mathrm{SO}(n)$ transformation which, in this new basis reads $\left(\begin{array}{cc}e^{-\mathrm{i} \beta} & 0 \\ 0 & e^{\mathrm{i} \beta}\end{array}\right)$. Taking into account that $\left(Z_{1}^{\prime}\right)^{2}+\left(Z_{2}^{\prime}\right)^{2}=2 e^{2 \mathrm{i} \varphi} \widetilde{\rho}_{1} \widetilde{\rho}_{2}, S_{1}$ and $S_{2}$ take the following form:

$$
S_{1}=z_{1}^{2}+z_{2}^{2}-\widetilde{\rho}_{1}^{2}-\widetilde{\rho}_{2}^{2} \quad S_{2}=2\left(z_{1} z_{2}-\widetilde{\rho}_{1} \widetilde{\rho}_{2} e^{2 \mathrm{i} \varphi}\right) .
$$


If we start from $Z_{I}=\rho_{I} e^{\mathrm{i} \theta_{I}}, I=1,2$, and fix $\theta_{1}-\theta_{2}=\pi / 2$, using the $\mathrm{SO}(2) \subset \mathrm{SO}(n)$ freedom, we easily find the following relations $\widetilde{\rho}_{1}=\frac{\rho_{1}+\rho_{2}}{\sqrt{2}}$ and $\widetilde{\rho}_{2}=\frac{\rho_{1}-\rho_{2}}{\sqrt{2}}$. The quartic invariant in this new normal form reads:

$$
I_{4}=\left(\widetilde{\rho}_{1}^{2}-\widetilde{\rho}_{2}^{2}\right)^{2}+\left(z_{1}^{2}-z_{2}^{2}\right)^{2}-2\left(\widetilde{\rho}_{1}^{2}+\widetilde{\rho}_{2}^{2}\right)\left(z_{1}^{2}+z_{2}^{2}\right)+8 \widetilde{\rho}_{1} \widetilde{\rho}_{2} z_{1} z_{2} \cos (2 \varphi)
$$

Notice the symmetry between $z_{1}, z_{2}$ and $\widetilde{\rho}_{1}, \widetilde{\rho}_{2}$. Indeed this normal form can be easily obtained from the $\mathcal{N}=8$ central charges by identifying $z_{1}, z_{2}, \widetilde{\rho}_{1}, \widetilde{\rho}_{2}$ with the moduli $\rho_{i}$ of the skew-eigenvalues of $Z_{A B}$.

It is straightforward to verify that for generic $\widetilde{\rho}_{1}, \widetilde{\rho}_{2}$ the stabilizer on the matter sector is $\mathrm{SO}(n-2)$. This does not change if either one or the other of the two norms is put to zero. If, on the other hand, $\widetilde{\rho}_{1}=\widetilde{\rho}_{2}$ the stabilizer is enhanced to $\mathrm{SO}(n-1)$ and, finally, if the two matter charges are both zero we recover the full $\mathrm{SO}(n)$. The five parameters $\left\{z_{1}, z_{2}, \tilde{\rho}_{1}, \tilde{\rho}_{2}, \varphi\right\}$ defining the normal form can be expressed in terms of the five $H$-invariant functions of the central and matter charges characterizing a generic configuration of scalar fields and charges at infinity, i.e. the $H$-orbit of the solution. The classification of small and large black holes, on the other hand, refers to the $G$-orbits of the quantized charges $\mathcal{P}$. Each $G$-orbit will in general comprise infinitely many $H$-orbits, defined by $G$-covariant conditions on the five invariant parameters.

\subsection{Large orbits}

Let us first consider the large black-hole solutions, with $I_{4} \neq 0$. We have three orbits, which can be characterized, all over the flow, in terms of the sign of $I_{4}$ and $S_{1}$ :

a) 1/4-BPS orbit, preserving the symmetry $\mathrm{SO}(4) \times \mathrm{SO}(\mathrm{n})$.

In this case $I_{4}>0$, and $S_{1}>0$.

$\beta)$ non-BPS orbit with $Z_{A B} \neq 0$, preserving $\mathrm{USp}(4) \times \mathrm{SO}(\mathrm{n}-1)$.

In this case $I_{4}<0$, and the sign of $S_{1}$ has no restrictions.

$\gamma$ ) non-BPS orbit with $Z_{A B}=0$, preserving the symmetry $\mathrm{SU}(4) \times \mathrm{SO}(\mathrm{n}-2)$.

In this case $I_{4}>0$, and $S_{1}<0$.

\subsection{Small orbits}

Let us now consider the case of small orbits, corresponding to $I_{4}=0$. They can be classified, in terms of the invariants introduced above, into 3 inequivalent classes [24]:

A) $T_{\Lambda \Sigma}=0$.

This class contains 3 different orbits, corresponding to $L^{0}$ being positive, negative or null:

A1) $1 / 2$-BPS orbit preserving the symmetry $\mathrm{USp}(4) \times \mathrm{SO}(\mathrm{n})$.

In this case $L^{0}, S_{1}>0$. 
A2) non-BPS orbit preserving the symmetry $\mathrm{SU}(4) \times \mathrm{SO}(\mathrm{n}-1)$.

In this case $L^{0}, S_{1}<0$.

A3) 1/2-BPS orbit preserving the symmetry $\mathrm{USp}(4) \times \mathrm{SO}(\mathrm{n}-1)$.

In this case $L^{\alpha}=0, S_{1}=S_{2}=0$.

We note that this class of orbits has a simple realization in the heterotic basis, where the charges $p_{\Lambda}=\mathcal{P}_{\Lambda}^{1}=0$. In this basis the three orbits correspond to the norm of $q_{\Lambda}$ being positive, negative, or null.

B) $T_{\Lambda \Sigma} \neq 0 ; L^{a}=0$.

This is a $1 / 4$-BPS orbit preserving the symmetry $\mathrm{SO}(4) \times \mathrm{SO}(\mathrm{n}-2)$.

C) $T_{\Lambda \Sigma} \neq 0 ; L^{a} \neq 0$.

It contains two orbits:

C1) $1 / 4$-BPS orbit preserving the symmetry $\mathrm{SO}(4) \times \mathrm{SO}(\mathrm{n}-1)$.

In this case $L^{0}, S_{1}>0$.

C2) non-BPS orbit preserving the symmetry $\operatorname{USp}(4) \times \mathrm{SO}(\mathrm{n}-2)$.

In this case $L^{0}, S_{1}<0$.

The above classification was found by studying the near-horizon properties of the solutions. However, since the different orbits are characterized in terms of U-duality invariants, actually the same properties hold true all over the flow of the fields from space infinity to the horizon.

\subsection{The $\mathcal{W}$ function for small orbits}

In the study of the large orbits of extremal black holes, the dynamical flow can be completely characterized in terms of the fake-superpotential $W$, which enjoys the property of being a monotonic function decreasing from space-infinity (at $\tau=0$ ) and to the horizon (at $\tau \rightarrow-\infty$ ). In particular the ADM mass is defined in terms of $W$ as:

$$
M_{A D M}=\lim _{\tau \rightarrow 0} \dot{U}=\lim _{\tau \rightarrow 0} \frac{1}{2} \mathcal{W}(\phi(\tau))=W\left(\phi_{0}\right),
$$

and it is bounded from below by the value taken at the horizon, which is fixed in terms of the charges

$$
M_{A D M}=W\left(\phi_{0}\right) \geq W(\phi(-\infty))=\sqrt{\frac{A_{H}}{4 \pi}} .
$$

In this respect small orbits are problematic because in this case the mass is not bounded from below. This raises the problem of defining the fake superpotential for small orbits, where the attractor mechanism breaks down. On the other hand, since $W$ is well defined for the large orbits, we may nevertheless try to define it from the large orbit cases by an appropriate limiting procedure obtained by choosing particular constraints equivalently on the bare or on the dressed charges, such that the horizon area collapses to zero:

$$
A_{H}=4 \pi \sqrt{\left|I_{4}\right|} \rightarrow 0 .
$$


This requires a careful analysis of the behavior under this limit of the invariants of the various orbits.

\subsection{Small versus large orbits}

In the following we give the constraints on the charges needed to obtain zero-horizon area for each of the orbits listed above. To this end let us describe the large orbits in terms of the five normal form parameters $z_{1}, z_{2}, \tilde{\rho}_{1}, \tilde{\rho}_{2}, \varphi$. In particular we shall consider representatives of the $G$-orbits of the quantized charges and the corresponding values of the five parameters on some specific point $\phi_{0}$ of the moduli space at infinity, keeping in mind that on a generic point the solution is characterized by five free parameters.

$\alpha)$ Being the solution 1/4-BPS, in a generic point $\phi_{0}$ at infinity the $W$ function is given by the highest eigenvalue of the central charge matrix, say $z_{1}$, expressed in terms of $\mathrm{H}$ invariant functions of the central and matter charges: $W=\left|z_{1}\right|$. As a representative of the orbit we can take $\tilde{\rho}_{2}=0$ and denote $\tilde{\rho}_{1}=\rho$. In this case $S_{2}$ is real and thus:

$$
I_{4}=\left(S_{1}-S_{2}\right)\left(S_{1}+S_{2}\right)=\left[\rho^{2}-\left(z_{1}+z_{2}\right)^{2}\right]\left[\rho^{2}-\left(z_{1}-z_{2}\right)^{2}\right]
$$

Requiring it to be positive together with $S_{1}$ leads to the condition $\rho^{2}<\left(z_{1}-z_{2}\right)^{2}$. As we shall see in the following, to obtain the $A$-type small orbits by setting some of the charges to zero, the above representative is not useful but a different one should be chosen, with $\tilde{\rho}_{1}=\tilde{\rho}_{2}$.

$\beta)$ We can choose $\tilde{\rho}_{1}=\tilde{\rho}_{2}=\rho$ and denote $z_{1}=z_{2}=z$. In this case we have:

$$
\begin{aligned}
S_{1} & =2\left(z^{2}-\rho^{2}\right), \\
S_{2} & =2\left(z^{2}-\rho^{2} e^{2 \mathrm{i} \varphi}\right), \\
I_{4} & =-8 \rho^{2} z^{2}(1-\cos (2 \varphi))<0 .
\end{aligned}
$$

For this orbit the general expression of $W$ in terms of $H$-invariant quantities is not known;

$\gamma$ ) The $W$ function for the $(\gamma)$ large orbit was computed in [2]. There it was shown that, fixing the relative phase of the two matter charges to $\pi / 2, W$ was simply expressed as $W=\frac{1}{\sqrt{2}}\left(\rho_{1}+\rho_{2}\right)=\tilde{\rho}_{1}$, no constraints being required on the invariant quantities $z_{1}, z_{2}, \tilde{\rho}_{I}, \varphi$ characterizing the solution at infinity. Just as in the $(\alpha)$ case, a representative of this $G$-orbit can be chosen by setting $\tilde{\rho}_{2}=0, \tilde{\rho}_{1}$ being denoted by $\rho$. The quartic invariant is given by eq. (4.15) and the condition $I_{4}>0, S_{1}<0$ requires taking $\rho^{2}>\left(z_{1}+z_{2}\right)^{2}$.

This representative, however, is not useful to retrieve the $A$-type small orbits by simple constraints on the remaining charges and a different representative should be chosen (having $\tilde{\rho}_{1}=\tilde{\rho}_{2}$ ).

The large orbit $(\beta)$ has no constraints on $S_{1}$, so it can generate all the small orbits by imposing appropriate relations among the charges. However, this orbit is the only one for 
which we do not have a complete knowledge of $W$, except for those $H$-orbits with fixed $\varphi$. We will then be interested in deriving the small orbits from simple conditions on the charges describing the $(\alpha)$ and $(\gamma)$ large ones, for which $W$ is known. The $W$ functions of the small orbits will then be obtained from those describing the $(\alpha)$ - and $(\gamma)$-solutions by imposing appropriate constraints on the quantized charges.

The $A$-type orbits. As pointed out earlier, the condition $T_{\Lambda \Sigma}=0$ can be solved by setting $p_{\Lambda}=0$. Under this condition, as shown in appendix $\mathrm{D}$, central and matter charges satisfy a reality condition, which, on the two normal forms, implies:

$$
\left\{z_{1}=z_{2}=z, \theta=0\right\} \Leftrightarrow\left\{z_{1}=z_{2}=z, \tilde{\rho}_{1}=\tilde{\rho}_{2}=\tilde{\rho}, \varphi=0\right\} .
$$

We can easily see from (4.11) that the above conditions imply $I_{4}=0$. In particular, the condition $\varphi \rightarrow 0$ implies

$$
I_{4} \rightarrow\left[\left(z_{1}-z_{2}\right)^{2}-\left(\tilde{\rho}_{1}-\tilde{\rho}_{2}\right)^{2}\right]\left[\left(z_{1}+z_{2}\right)^{2}-\left(\tilde{\rho}_{1}+\tilde{\rho}_{2}\right)^{2}\right]
$$

A1) Since this orbit has $S_{1}>0$, it can be derived from the large orbits $(\alpha)$ and $(\beta)$.

The orbit $(\alpha)$ in the normal form has:

$$
z_{1}^{2}+z_{2}^{2}>\tilde{\rho}_{1}^{2}+\tilde{\rho}_{2}^{2} .
$$

To obtain the $G$-orbit $(A 1)$ from the $(\alpha)$-orbit it suffices to set $z_{1} \rightarrow z_{2}, \tilde{\rho}_{1}=\tilde{\rho}_{2}=0$ consistently with (4.17). Then from (4.18) $I_{4} \rightarrow 0$. The compact symmetry of the charges is enhanced to $H_{0}=\mathrm{USp}(4) \times \mathrm{SO}(\mathrm{n})$ and the preserved amount of supersymmetry is doubled to $1 / 2$-BPS.

The $W$ function is given, in a generic point on the moduli space at infinity, by the highest eigenvalue of the central charge matrix: $W=z_{1}$;

A2) It has $S_{1}<0$, so that it can be obtained from orbits $(\beta)$ and $(\gamma)$. This is consistent with the fact that it is a non-BPS orbit, which cannot be obtained from a BPS one by imposing relations on the charges. The orbit $(\gamma)$ in the normal form has:

$$
z_{1}^{2}+z_{2}^{2}<\tilde{\rho}_{1}^{2}+\tilde{\rho}_{2}^{2} .
$$

To obtain a representative of the $G$-orbit $(A 2)$ from $(\gamma)$ we can start from a representative of the latter with $z_{1}=z_{2}=0$ and impose condition (4.17) which requires setting $\tilde{\rho}_{1}=\tilde{\rho}_{2}$.

The compact stabilizer of the charges is enlarged, with respect to the original orbit $(\gamma)$, to $H_{0}=\mathrm{SU}(4) \times \mathrm{SO}(\mathrm{n}-1)$.

The $W$ function is given, in a generic point on the moduli space at infinity, by the highest of the two matter charge moduli $\tilde{\rho}_{I}: W=\tilde{\rho}_{1}$;

A3) This $A$-orbit has $S_{1}=0$. It can be obtained from all the large orbits $(\alpha),(\beta)$ and $(\gamma)$. In particular, it is found from orbits $(\alpha)$ and $(\gamma)$, by imposing (4.17), so that in particular $S_{1}$ reduces to

$$
S_{1}=2\left(z^{2}-\tilde{\rho}^{2}\right)
$$


supplemented by the condition

$$
z^{2}=\tilde{\rho}^{2}
$$

In this limit the compact stabilizer of quantized charges reduces to $H_{0}=\mathrm{USp}(4) \times$ $\mathrm{SO}(\mathrm{n}-1)$. Being the solution 1/2-BPS, the $W$ function is given, in a generic point on the moduli space at infinity, by the highest eigenvalue of the central charge matrix: $W=z_{1}$. Note that eq. (4.22) is a condition between central and matter charges, and it is a necessary condition, when the orbit is obtained from the $(\gamma)$ large orbit, for the enhancement of supersymmetry from non-BPS to 1/2-BPS, since for the $(\gamma)$ orbit $W$ is given by the highest eigenvalue of the matter charges, while in the BPS cases it is given by the highest eigenvalue of the central charges.

The $B$ and $C$ type orbits. These orbits are characterized by having $T_{\Lambda \Sigma} \neq 0$. This implies that some of the conditions (4.17) should be relaxed. In particular we could have the following possibilities:

$$
\begin{aligned}
B): z_{1} \neq z_{2}, & \tilde{\rho}_{1} \neq \tilde{\rho}_{2}, \\
C 1): z_{1} \neq z_{2}, & \tilde{\rho}_{1}=\tilde{\rho}_{2}, \\
C 2): z_{1}=z_{2}, & \tilde{\rho}_{1} \neq \tilde{\rho}_{2} .
\end{aligned}
$$

Let examine these three cases in some detail.

B) The whole vector $L_{1}{ }^{a}$ is zero in this case, which implies $S_{1}=S_{2}=0$. We can start from a representative of either the $(\alpha)$ or the $(\gamma)$-orbit with $z_{2}=0=\tilde{\rho_{2}}$. In the former case $z_{1}^{2}>\tilde{\rho}_{1}^{2}$ while in the latter $z_{1}^{2}<\tilde{\rho}_{1}^{2}$, while $S_{1}$ and $I_{4}$ read:

$$
\begin{gathered}
S_{1}=z_{1}^{2}-\tilde{\rho}_{1}^{2}, \quad S_{2}=0, \\
I_{4}=S_{1}^{2}=\left(z_{1}^{2}-\tilde{\rho}_{1}^{2}\right)^{2} .
\end{gathered}
$$

To obtain a representative of the $B$-orbit we need to set:

$$
z_{1}^{2}=\tilde{\rho}_{1}^{2}
$$

The compact little group of this orbit is $H_{0}=\mathrm{SO}(4) \times \mathrm{SO}(\mathrm{n}-2)$, and, being the solution 1/4-BPS, the $W$ function on a generic point of the moduli space is given by the highest eigenvalue of the central charge matrix, say $W=z_{1}$. Similarly to the (A3) case, we note that the condition (4.25) between central and matter charges is necessary, when the orbit is obtained from the $(\gamma)$ large orbit, for the enhancement of supersymmetry from non-BPS to 1/4-BPS.

C1) It has $S_{1}>0$ so it can be obtained from orbits $(\alpha),(\beta)$. We can start from a representative of $(\alpha)$ with $\varphi=0$ and $\tilde{\rho}_{1}=\tilde{\rho}_{2}=\tilde{\rho}$ (but $z_{1}>z_{2}$ ). In this case

$$
I_{4}=\left(z_{1}-z_{2}\right)^{2}\left[\left(z_{1}+z_{2}\right)^{2}-4 \tilde{\rho}^{2}\right] \geq 0 .
$$

If we further impose $4 \tilde{\rho}^{2}=\left(z_{1}+z_{2}\right)^{2}$, we find $I_{4}=0$ and $S_{1}=\frac{1}{2}\left(z_{1}-z_{2}\right)^{2} \geq 0$. The compact little group of this $G$-orbit is $H_{0}=\mathrm{SO}(4) \times \mathrm{SO}(\mathrm{n}-1)$ and, being it $1 / 4$-BPS, the $W$ function is given, in a generic point on the moduli space at infinity, by the highest eigenvalue of the central charge matrix: $W=z_{1}$. 
C2) It has $S_{1}<0$ so it can be obtained from the orbits $(\beta),(\gamma)$. In the latter case we can start from a representative with $z_{1}=z_{2}=z, \tilde{\rho}_{2}=0$ and $\tilde{\rho}=\tilde{\rho}_{1} \geq 4 z^{2}$. In this case $S_{1}$ and $I_{4}$ read:

$$
S_{1}=2 z^{2}-\tilde{\rho}^{2} \leq 0 \quad, \quad I_{4}=\tilde{\rho}^{2}\left(\tilde{\rho}^{2}-4 z^{2}\right)>0 .
$$

A representative of $C 2$ is obtained by setting $\tilde{\rho}^{2}=4 z^{2}$.

On a generic point in the moduli space, the $W$ function is given by the highest of the two matter charge moduli $\tilde{\rho}_{I}$, say $W=\tilde{\rho}_{1}$.

\section{Small black holes in $\mathcal{N}=2$ magic models}

We are going to show that, as in the $\mathcal{N}=4$ case, the $\mathcal{N}=2$ ADM mass for small black holes either is supersymmetric (and then given by $\left|Z\left(\phi_{0}\right)\right|$, in terms of the asymptotic value at $\tau \rightarrow 0$ of the central charge) or can be obtained by a non-BPS black hole with $Z=0$, and it has therefore a known expression in terms of radicals [6, 25]. An exceptional case in this respect is the $t^{3}$-model which has no $Z=0$ orbit. In this case, as we shall show below, the $W$ for the small orbit can be obtained either from that of the BPS $I_{4}>0$ orbit or from the $W$-function of the non-BPS $I_{4}<0$ orbit, which is known [4].

\section{1 $D=6$ uplift of magic models}

In $D=6$ there are three types of black holes, corresponding to the sign of the quadratic form

$$
X^{2}=X^{A} \eta_{A B} X^{B}
$$

written in terms of the scalars in the tensor multiplets $X^{A} \in \mathrm{SO}(q+1,1) / \mathrm{SO}(q+1)$ ( $q=1,2,4,8$ for real, complex, quaternionic o octonionic models respectively). In particular, for $X^{2} \neq 0$ we have large black holes, associated to two-charge solutions, BPS for $X^{2}>0$, generated by two quantized charges of the same sign, and non-BPS for $X^{2}<0$, corresponding to quantized charges of opposite sign. For $X^{2}=0$ we have instead a small black hole, which is a BPS one-charge solution.

The compact symmetry of the three different orbits are

- $\mathrm{SO}(\mathrm{q}+1) \quad$ for the BPS large black hole

- $\mathrm{SO}(\mathrm{q}) \quad$ for the non-BPS large black hole

- $\mathrm{SO}(\mathrm{q}) \quad$ for the BPS small black hole

We are going to consider in the following the octonionic case $(q=8)$.

\section{$5.2 D=5$ uplift}

There are three orbits of small black holes at $D=5$, corresponding to the vanishing of the cubic invariant $I_{3}=0$. Two of them are two-charge configurations, BPS if the 2 charges have the same sign, non-BPS otherwise, while the third is a BPS one-charge configuration, 
corresponding to $I_{3}=\partial I_{3}=0$. These three orbits are the trivial dimensional reduction of the six-dimensional orbits. They preserve the compact symmetries $\mathrm{SO}(9), \mathrm{SO}(8)$ and $\mathrm{SO}(9)$ respectively.

Moreover, there are two large orbits corresponding to three-charge configurations (with $I_{3} \neq 0$ ), a BPS one for charges of the same sign, and a non-BPS one, when one of the charges has opposite sign with respect to the other two. The compact symmetries preserved are $F_{4}$ and $\mathrm{SO}(9)$ respectively.

\section{3 $D=4$ analysis}

At $D=4$ there are five small orbits. Three of them originate from dimensional reduction of the small $D=5$ orbits, and have compact symmetry $\mathrm{O}(10), \mathrm{O}(9) \times \mathrm{O}(2)$ (critical, two charges) and $\mathrm{F}_{4}$ (double critical, one charge); two of them are three-charge light-like orbits coming by direct dimensional reduction of the large $D=5$ orbits and have compact symmetries $\mathrm{F}_{4}$ and $\mathrm{SO}(9)$.

\subsection{ADM mass for small black holes at $D=4$}

For the three BPS orbits it is given by the asymptotic value, at space infinity $(\tau \rightarrow 0)$ of the norm of the central charge $Z$, at $I_{4}=0$. For the two non-BPS orbits, both can be obtained by a large non-BPS $Z=0$ orbit (with charges $(+,+,-,-)$ ) by setting one of the charges to zero. Then the ADM mass is given by the asymptotic limit of $W$, which is obtained from the one of the corresponding large orbit, which is a known radical function of the H-invariants (see eqs. (5.11) and (5.12) of [6] and eq. (3.4) of [5]).

\subsection{The $W$ function of the small black holes in the $t^{3}$-Model}

As anticipated, the $t^{3}$-model deserves a spacial treatment in our analysis since it does not have the non-BPS orbit with $Z=0$. However, in this case, both the $W$ functions for the $I_{4}>0$ (BPS) and the $I_{4}<0$ (non-BPS) orbits are known. The former is $W=\sqrt{i_{1}}=|Z|$, while the latter is given in eq. (3.15) of [4] and reads:

$$
\begin{aligned}
W^{2}= & \frac{i_{1}+i_{2}}{4}+\frac{3}{8}\left[\left(\left(i_{1}-\frac{i_{2}}{3}\right)^{3}-\left(i_{1}+i_{2}\right) I_{4}+4 i_{3} \sqrt{-I_{4}}\right)^{1 / 3}+\right. \\
& \left.+\left(\left(i_{1}-\frac{i_{2}}{3}\right)^{3}-\left(i_{1}+i_{2}\right) I_{4}-4 i_{3} \sqrt{-I_{4}}\right)^{1 / 3}\right]
\end{aligned}
$$

It is straightforward to verify that the above expression has a finite $I_{4} \rightarrow 0$ limit which yields $W=\sqrt{i_{1}}=|Z|$. In the $I_{4} \rightarrow 0$ limit therefore, both the $W$-functions associated with the two large black hole orbits coincide with the modulus of the central charge, as expected since small black holes in this model are BPS.

\section{A Proof of eq. (2.12)}

Equation (2.12), as shown in [3], is a particular form of the general solution to the HamiltonJacobi equation. In what follows we shall tailor the formal proof given in [3] to the class 
of extremal solutions we are considering, without making use of the Hamilton-Jacobi formalism.

Consider the extremal solutions $U\left(\tau ; \phi_{0}, U_{0}\right)$ and $\phi\left(\tau ; \phi_{0}, U_{0}\right)$, for a given charge vector $\mathcal{P}$, within the interval $\tau_{*}<\tau<\tau_{0}$, where now $U_{0}, \phi_{0}$ denote the values of the fields computed at $\tau_{0}: U_{0}=U\left(\tau_{0} ; \phi_{0}, U_{0}\right), \phi_{0}=\phi\left(\tau_{0} ; \phi_{0}, U_{0}\right)$. The values of the fields at $\tau_{*}$, for our family of solutions, is completely fixed in terms of $\left(U_{0}, \phi_{0}\right)$ and $\mathcal{P}$. Let us perform an infinitesimal variation of the boundary conditions: $U_{0} \rightarrow U_{0}+\delta U_{0}$ and $\phi_{0}=\phi_{0}+\delta \phi_{0}$. This will determine a new solution within the same class:

$$
\begin{aligned}
U\left(\tau ; \phi_{0}+\delta \phi_{0}, U_{0}+\delta U_{0}\right) & =U\left(\tau ; \phi_{0}, U_{0}\right)+\delta U(\tau), \\
\phi\left(\tau ; \phi_{0}+\delta \phi_{0}, U_{0}+\delta U_{0}\right) & =\phi\left(\tau ; \phi_{0}, U_{0}\right)+\delta \phi(\tau) .
\end{aligned}
$$

Now we write a seemingly more general ansatz for $W$ than the one in eq. (2.12):

$$
e^{U_{0}} W\left(\phi_{0}, \mathcal{P}\right)=e^{U_{*}} W\left(\phi_{*}, \mathcal{P}\right)+\int_{\tau_{*}}^{\tau_{0}} e^{2 U\left(\tau ; U_{0}, \phi_{0}\right)} V\left(\phi\left(\tau ; U_{0}, \phi_{0}\right), \mathcal{P}\right) d \tau
$$

As we shall see, the result of this integral does not depend on the choice of $\tau_{0}$. For the sake of simplicity we shall suppress the dependence on $\tau$ and on the boundary values of the fields in the integrand. Since the integral is computed along solutions, we can use the Hamiltonian constraint (2.5) to rewrite $W$ as follows:

$$
\begin{aligned}
e^{U_{0}} W\left(\phi_{0}, \mathcal{P}\right) & =e^{U_{*}} W\left(\phi_{*}, \mathcal{P}\right)+\frac{1}{2} \int_{\tau_{*}}^{\tau_{0}}\left[e^{2 U} V(\phi, \mathcal{P})+\dot{U}^{2}+\frac{1}{2} G_{r s} \dot{\phi}^{r} \dot{\phi}^{s}\right] d \tau= \\
& =e^{U_{*}} W\left(\phi_{*}, \mathcal{P}\right)+\frac{1}{2} \int_{\tau_{*}}^{\tau_{0}} \mathcal{L}_{\mathrm{eff}}(U, \phi, \dot{U}, \dot{\phi}) d \tau
\end{aligned}
$$

Now perform the variation (A.1), integrate by parts and use the equations of motion:

$$
\begin{aligned}
& \delta U_{0} e^{U_{0}} W\left(\phi_{0}, \mathcal{P}\right)+e^{U_{0}} \partial_{r} W\left(\phi_{0}, \mathcal{P}\right) \delta \phi_{0}^{r}=\delta\left(e^{U_{*}} W\left(\phi_{*}, \mathcal{P}\right)\right)+ \\
& \quad+\frac{1}{2} \int_{\tau_{*}}^{\tau_{0}}\left[\left(\frac{\partial}{\partial U} \mathcal{L}_{\mathrm{eff}}-\frac{d}{d \tau} \frac{\partial}{\partial \dot{U}} \mathcal{L}_{\mathrm{eff}}\right) \delta U+\left(\frac{\partial}{\partial \phi^{r}} \mathcal{L}_{\mathrm{eff}}-\frac{d}{d \tau} \frac{\partial}{\partial \dot{\phi}^{r}} \mathcal{L}_{\mathrm{eff}}\right) \delta \phi^{r}\right]+ \\
& +\left.\left(\dot{U} \delta U+\frac{1}{2} G_{r s} \dot{\phi}^{s} \delta \phi^{r}\right)\right|_{\tau_{*}} ^{\tau_{0}}=\delta\left(e^{U_{*}} W\left(\phi_{*}, \mathcal{P}\right)\right)+\left.\left(\dot{U} \delta U+\frac{1}{2} G_{r s} \dot{\phi}^{s} \delta \phi^{r}\right)\right|_{\tau_{*}} ^{\tau_{0}}
\end{aligned}
$$

where we have used the short-hand notation $\partial_{r} W \equiv \frac{\partial W}{\partial \phi^{r}}$. We can choose $\tau_{*}=-\infty$, so that all terms computed at $\tau_{*}$ in the above equation vanish. Equating the variations at $\tau_{0}$ on both sides we find:

$$
\dot{U}\left(\tau_{0}\right)=e^{U_{0}} W\left(\phi_{0}, \mathcal{P}\right), \quad \dot{\phi}^{s}\left(\tau_{0}\right)=2 e^{U_{0}} G^{r s}\left(\phi_{0}\right) \partial_{r} W\left(\phi_{0}, \mathcal{P}\right) .
$$

Being $\tau_{0}$ generic, we find that $W$ defines the first order equations (2.6) for the fields and thus it is a solution to the Hamilton-Jacobi equation.

Note however that $W$, as defined in (A.2), may in principle depend on the chosen value of $\tau_{0}$, that is $W=W\left(U_{0}, \phi_{0}, \tau_{0}, \mathcal{P}\right)$. Let us show that this is not the case, namely that $W\left(U_{0}, \phi_{0}, \tau_{0}+\delta \tau, \mathcal{P}\right)=W\left(U_{0}, \phi_{0}, \tau_{0}, \mathcal{P}\right)$, for a generic $\delta \tau$. To do this we vary $\tau_{0} \rightarrow \tau_{0}+\delta \tau$, 
keeping the boundary values of the fields fixed. This requires to change the solution on which the integral is computed from $U(\tau), \phi(\tau)$ to $U^{\prime}(\tau), \phi^{\prime}(\tau)$ such that:

$$
\begin{aligned}
U^{\prime}\left(\tau_{0}+\delta \tau\right) & =U\left(\tau_{0}\right)=U\left(\tau_{0}+\delta \tau\right)-\dot{U}\left(\tau_{0}\right) \delta \tau, \\
\phi^{\prime}\left(\tau_{0}+\delta \tau\right) & =\phi\left(\tau_{0}\right)=\phi\left(\tau_{0}+\delta \tau\right)-\dot{\phi}\left(\tau_{0}\right) \delta \tau .
\end{aligned}
$$

and thus amounts to performing, along the flow, the transformation $U \rightarrow U-\dot{U} \delta \tau, \phi \rightarrow$ $\phi-\dot{\phi} \delta \tau$, besides changing the domain of integration, $\delta \tau$ being chosen along the flow so that $\delta \tau_{*}=0$. After some straightforward calculations we find:

$$
e^{U_{0}}\left(W\left(U_{0}, \phi_{0}, \tau_{0}+\delta \tau, \mathcal{P}\right)-W\left(U_{0}, \phi_{0}, \tau_{0}, \mathcal{P}\right)\right)=\left.\mathcal{H}_{\mathrm{eff}}\right|_{\tau_{0}} \delta \tau=0,
$$

in virtue of the Hamiltonian constraint. Since the function $W$ of the moduli space, as defined by (A.2), does not depend on the choice of $\tau_{0}$, we can choose $\tau_{0}=0$, where $U_{0}=0$ and then find (2.12).

\section{B Stability and asymptotic stability in the sense of Liapunov}

Let us briefly recall the notion of stability (in the sense of Liapunov) and of attractiveness of an equilibrium point. Given an autonomous dynamical system:

$$
\dot{\phi}^{r}=f^{r}(\phi),
$$

an equilibrium point $\phi_{*}\left(f^{r}\left(\phi_{*}\right)=0\right.$ ), is attractive (or an attractor), for $\tau \rightarrow-\infty$, if there exist a neighborhood $\mathcal{I}_{\phi_{*}}$ of $\phi_{*}$, such that all trajectories $\phi^{r}\left(\tau, \phi_{0}\right)$ originating at $\tau=0$ in $\phi_{0} \in \mathcal{I}_{\phi_{*}}$ evolve towards $\phi_{*}$ as $\tau \rightarrow-\infty$ :

$$
\lim _{\tau \rightarrow-\infty} \phi^{r}\left(\tau, \phi_{0}\right)=\phi_{*}^{r}, \quad \forall \phi_{0} \in \mathcal{I}_{\phi_{*}} .
$$

An equilibrium point $\phi_{*}$ (not necessarily attractive) is stable (in the sense of Liapunov) if, for any $\epsilon>0$, there exist a ball $\mathcal{B}_{\delta}\left(\phi_{*}\right)$ of radius $\delta>0$ centered in $\phi_{*}$, such that:

$$
\forall \phi_{0} \in \mathcal{B}_{\delta}\left(\phi_{*}\right), \quad \forall \tau<0: \phi\left(\tau, \phi_{0}\right) \in \mathcal{B}_{\epsilon}\left(\phi_{*}\right),
$$

that is, provided we take the starting point $\phi_{0}$ sufficiently close to $\phi_{*}$, the entire solution will stay, for all $\tau<0$, in any given, whatever small, neighborhood of $\phi_{*}$. Finally an equilibrium point is asymptotically stable (in the sense of Liapunov) if it is attractive and stable.

Liapunov's Theorem: If there exist a function $v(\phi)$ which is positive definite in a neighborhood of $\phi_{*}$ (that is positive in a neighborhood of $\phi_{*}$ and $v\left(\phi_{*}\right)=0$ ) and such that also the derivative of $v$ along the solution, in the same neighborhood, is positive definite: ${ }^{9}$ $\frac{d v}{d \tau}=\dot{\phi}^{r} \partial_{r} v>0$, then $\phi_{*}$ is an asymptotically stable equilibrium point or, equivalently, a stable attractor.

For large extremal black holes such function is $v(\phi)=W(\phi)-W\left(\phi_{*}\right)=W(\phi)-\sqrt{\left|I_{4}\right|}$.

\footnotetext{
${ }^{9}$ Here we require positive definiteness because our critical point is located at $\tau \rightarrow-\infty$ and not at $+\infty$ as in standard textbooks.
} 


\section{Properties of the Vielbein on $\mathscr{M}_{\text {scal }}$}

Let us briefly motivate why, for all regular extremal black holes, our choice of parametrization is such that the vielbein matrix has a vanishing off-diagonal block $V_{k}{ }^{a}$.

The reason is purely group theoretical. As far as the BPS and non-BPS $\left(I_{4}>0\right)$ orbits are concerned, taking into account that $\phi^{k}$ belong to $\mathbf{R}_{1}$ and the index $a$ label the $\mathbf{R}_{0}$ representation, $d \phi^{k} V_{k}{ }^{a}\left(\phi^{k}\right)$ can be different from zero only if $\mathbf{R}_{0}$ is contained in the tensor product of a number of $\mathbf{R}_{1}$ representations. As the reader can ascertain from table 1, this is never the case. For example in the case of regular BPS black holes, for $\mathcal{N}>2, \mathbf{R}_{0}$ is a doublet with respect to an $\mathrm{SU}(2)$ subgroup of $H_{0}$, while $\mathbf{R}_{1}$ is a singlet with respect to the same group. If we think of the $\mathcal{N}=2$ truncation of the original theory of which the same black hole is a $1 / 2$-BPS solution, this $\mathrm{SU}(2)$ group is the quaternionic structure of a quaternionic Kähler submanifold of the scalar manifold spanned by the scalars $\phi^{\alpha}$. On the other hand, as far as the non-BPS solutions with $I_{4}<0$ are concerned, the above argument does not apply in the coset parametrization (2.45), but choosing instead the solvable parametrization one finds $d \phi^{k} V_{k}{ }^{a}\left(\phi^{k}\right)=0$ since $\mathbb{L}_{1}{ }^{-1} d \mathbb{L}_{1}$ belongs to the same solvable algebra spanned by $\phi^{k}$, which is orthogonal to $\mathfrak{K}_{0}$.

\section{An explicit parametrization of the $\mathcal{N}=4$ scalar manifold}

The quantized charges $\mathcal{P}=\left(\mathcal{P}^{M}\right)$ of the $\mathcal{N}=4$ model transform under $G=\operatorname{SL}(2, \mathbb{R}) \times$ $\mathrm{SO}(6, \mathrm{n})$ in the representation $(\mathbf{2}, \mathbf{6}+\mathbf{n})$, which can be labeled by the couple of indices $M=(\alpha, \Lambda), \alpha=1,2, \Lambda=1, \ldots, 6+n$. In this basis the symplectic invariant matrix reads:

$$
\mathbb{C}_{M N}=\mathbb{C}_{\alpha \Lambda, \beta \Sigma}=-\epsilon_{\alpha \beta} \eta_{\Lambda \Sigma},
$$

Identifying the magnetic and electric charges with the components: $\mathcal{P}^{1 \Lambda}=p^{\Lambda}, \mathcal{P}^{2 \Lambda}=$ $q^{\Lambda} \equiv \eta^{\Lambda \Sigma} q_{\Sigma}$, corresponds to choosing the symplectic frame originating from the toroidal compactification of the heterotic string, in which $\mathrm{SO}(6, \mathrm{n})$ acts block-diagonally. We shall denote by $L_{2}=L_{2}{ }^{\Lambda}{ }_{\Sigma}$ the coset representative of $\frac{\mathrm{SO}(6, \mathrm{n})}{\mathrm{SO}(6) \times \mathrm{SO}(\mathrm{n})}$ and by $L_{1}=L_{1}{ }^{\alpha}{ }_{\beta}$ that of $\frac{\mathrm{SL}(2, \mathbb{R})}{\mathrm{SO}(2)}$, defined as:

$$
L_{1}{ }^{\alpha}{ }_{\beta}=\left(\begin{array}{cc}
e^{-\frac{\phi}{2}} & 0 \\
-A e^{-\frac{\phi}{2}} & e^{\frac{\phi}{2}}
\end{array}\right)
$$

where $S=A-\mathrm{i} e^{\phi}$ is the complex scalar parametrizing the lower-half plane $\frac{\mathrm{SL}(2, \mathbb{R})}{\mathrm{SO}(2)}$.

Using the definition (2.36) of the central and matter charges, we can write $Z_{\hat{M}}$ as $Z_{a \Lambda}$, where $a=1,2$ labels the complex basis in which $\mathrm{SO}(2)$ acts diagonally and which is obtained from the real one through the action of the Cayley matrix $\mathcal{A}=\left(\mathcal{A}_{a}{ }^{\beta}\right)=$ $\frac{1}{\sqrt{2}}\left(\begin{array}{cc}1 & \mathrm{i} \\ 1 & -\mathrm{i}\end{array}\right)$. In this notation $Z_{\hat{1} \Lambda}=\left(Z_{r}, Z_{I}\right)$, where $Z_{r}=\frac{1}{2} \Gamma_{r}{ }^{A B} Z_{A B}, r=1, \ldots, 6$, are the central charges and $Z_{I}, I=1, \ldots, n$, are the matter ones. We find:

$$
Z_{a \Lambda}=\mathcal{A}_{a}^{\beta} L_{1}^{\sigma}{ }_{\beta} L_{2}{ }^{\Gamma}{ }_{\Lambda} \epsilon_{\sigma \delta} \mathcal{P}_{\Gamma}^{\delta},
$$


Using the explicit parametrization (D.2) we find:

$$
Z_{\hat{1} \Lambda}=\left(Z_{r}, Z_{I}\right)=\frac{1}{\sqrt{-2 \operatorname{Im}(S)}} L_{2}{ }^{\Gamma} \Lambda\left(q_{\Gamma}+S p_{\Gamma}\right) .
$$

We see that $\left(Z_{r}, Z_{I}\right)$ are real iff $p^{\Lambda}=0$. Reality of the $Z_{r}$ implies the following reality condition on the $Z_{A B}$ :

$$
Z_{A B}=\frac{1}{2} \epsilon_{A B C D} \bar{Z}^{C D}
$$

If $Z_{A B}$ is in the normal form, the above condition implies $z_{1}=z_{2}$. Similarly, if the non vanishing matter charges $Z_{I=1,2}=\rho_{I}$, characterizing the normal form, are real, from (4.9) we find that $Z_{1}^{\prime}=\frac{1}{\sqrt{2}}\left(\rho_{1}+\mathrm{i} \rho_{2}\right)=\tilde{\rho}_{1} e^{\mathrm{i} \beta}, Z_{2}^{\prime}=\overline{Z_{1}^{\prime}}=\tilde{\rho}_{2} e^{-\mathrm{i} \beta}$, and thus that $\tilde{\rho}_{1}=\tilde{\rho}_{2}$.

\section{Acknowledgments}

The Work of L.A., R.D. and M.T. is supported in part by PRIN Program 2007 of MIUR and by INFN, sez. Torino. The work of S.F. is supported by ERC Advanced Grant n.226455, "Supersymmetry, Quantum Gravity and Gauge Fields" (Superfields), in part by PRIN 2007-0240045 of Torino Politecnico, in part by DOE Grant DE-FG03-91ER40662 and in part by INFN, sez. L.N.F.

Open Access. This article is distributed under the terms of the Creative Commons Attribution Noncommercial License which permits any noncommercial use, distribution, and reproduction in any medium, provided the original author(s) and source are credited.

\section{References}

[1] A. Ceresole and G. Dall'Agata, Flow Equations for Non-BPS Extremal Black Holes, JHEP 03 (2007) 110 [hep-th/0702088] [SPIRES].

[2] L. Andrianopoli, R. D'Auria, E. Orazi and M. Trigiante, First Order Description of Black Holes in Moduli Space, JHEP 11 (2007) 032 [arXiv:0706.0712] [SPIRES].

[3] L. Andrianopoli, R. D'Auria, E. Orazi and M. Trigiante, First Order Description of D $=4$ static Black Holes and the Hamilton-Jacobi equation, Nucl. Phys. B 833 (2010) 1 [arXiv:0905.3938] [SPIRES].

[4] A. Ceresole, G. Dall'Agata, S. Ferrara and A. Yeranyan, First order flows for $N=2$ extremal black holes and duality invariants, Nucl. Phys. B 824 (2010) 239 [arXiv:0908.1110] [SPIRES].

[5] G. Bossard, Y. Michel and B. Pioline, Extremal black holes, nilpotent orbits and the true fake superpotential, JHEP 01 (2010) 038 [arXiv: 0908.1742] [SPIRES].

[6] A. Ceresole, G. Dall'Agata, S. Ferrara and A. Yeranyan, Universality of the superpotential for $D=4$ extremal black holes, arXiv:0910.2697 [SPIRES].

[7] D.Z. Freedman, C. Núñez, M. Schnabl and K. Skenderis, Fake Supergravity and Domain Wall Stability, Phys. Rev. D 69 (2004) 104027 [hep-th/0312055] [SPIRES]. 
[8] S. Bellucci, S. Ferrara, M. Günaydin and A. Marrani, Charge orbits of symmetric special geometries and attractors, Int. J. Mod. Phys. A 21 (2006) 5043 [hep-th/0606209] [SPIRES].

[9] S. Bellucci, S. Ferrara, R. Kallosh and A. Marrani, Extremal Black Hole and Flux Vacua Attractors, Lect. Notes Phys. 755 (2008) 115 [arXiv:0711.4547] [SPIRES].

[10] V.I. Arnold, Mathematical Methods of Classical Mechanics, Graduate Texts in Mathematics, Springer, (1997).

[11] K. Meyer, G. Hall, Introduction to Hamiltonian Dynamical Systems and the N-Body Problem, Springer-Verlag, New York U.S.A. (1992).

[12] J. de Boer, E.P. Verlinde and H.L. Verlinde, On the holographic renormalization group, JHEP 08 (2000) 003 [hep-th/9912012] [SPIRES].

[13] E.P. Verlinde and H.L. Verlinde, RG-flow, gravity and the cosmological constant, JHEP 05 (2000) 034 [hep-th/9912018] [SPIRES].

[14] M. Fukuma, S. Matsuura and T. Sakai, Holographic renormalization group, Prog. Theor. Phys. 109 (2003) 489 [hep-th/0212314] [SPIRES].

[15] K. Skenderis and P.K. Townsend, Hamilton-Jacobi method for Domain Walls and Cosmologies, Phys. Rev. D 74 (2006) 125008 [hep-th/0609056] [SPIRES].

[16] P.K. Townsend, Hamilton-Jacobi Mechanics from Pseudo-Supersymmetry, Class. Quant. Grav. 25 (2008) 045017 [arXiv: 0710.5178] [SPIRES].

[17] K. Hotta, Holographic RG flow dual to attractor flow in extremal black holes, Phys. Rev. D 79 (2009) 104018 [arXiv:0902.3529] [SPIRES].

[18] B. Janssen, P. Smyth, T. Van Riet and B. Vercnocke, A first-order formalism for timelike and spacelike brane solutions, JHEP 04 (2008) 007 [arXiv:0712.2808] [SPIRES].

[19] W. Hahn, Stability of Motion, Springer-Verlag, Berlin Germany (1967).

[20] N. Rouche and J. Mawhin, Ordinary Differential Equations. Stability and Periodic Solutions, Pitman, Boston/London/Melbourne, (1980).

[21] S. Ferrara and A. Marrani, On the Moduli Space of non-BPS Attractors for $N=2$ Symmetric Manifolds, Phys. Lett. B 652 (2007) 111 [arXiv:0706.1667] [SPIRES].

[22] M. Bianchi, S. Ferrara and R. Kallosh, Perturbative and Non-perturbative $N=8$ Supergravity, Phys. Lett. B 690 (2010) 328 [arXiv:0910.3674] [SPIRES].

[23] M. Bianchi, S. Ferrara and R. Kallosh, Observations on Arithmetic Invariants and U-duality Orbits in $N=8$ Supergravity, JHEP 03 (2010) 081 [arXiv:0912.0057] [SPIRES].

[24] B.L. Cerchiai, S. Ferrara, A. Marrani and B. Zumino, Duality, Entropy and ADM Mass in Supergravity, Phys. Rev. D 79 (2009) 125010 [arXiv:0902.3973] [SPIRES].

[25] L. Andrianopoli, S. Ferrara, A. Marrani and M. Trigiante, Non-BPS Attractors in $5 d$ and $6 d$ Extended Supergravity, Nucl. Phys. B 795 (2008) 428 [arXiv:0709.3488] [SPIRES].

[26] L. Andrianopoli, R. D'Auria, S. Ferrara and M. Trigiante, Extremal black holes in supergravity, Lect. Notes Phys. 737 (2008) 661 [hep-th/0611345] [SPIRES].

[27] P. Aschieri, S. Ferrara and B. Zumino, Duality Rotations in Nonlinear Electrodynamics and in Extended Supergravity, Riv. Nuovo Cim. 31 (2008) 625 [arXiv:0807.4039] [SPIRES].

[28] S. Bellucci, S. Ferrara, M. Günaydin and A. Marrani, SAM Lectures on Extremal Black Holes in $D=4$ Extended Supergravity, arXiv:0905.3739 [SPIRES]. 
[29] E.G. Gimon, F. Larsen and J. Simon, Black Holes in Supergravity: the non-BPS Branch, JHEP 01 (2008) 040 [arXiv:0710.4967] [SPIRES].

[30] E. Cremmer and B. Julia, The SO(8) Supergravity, Nucl. Phys. B 159 (1979) 141 [SPIRES].

[31] R. Kallosh and B. Kol, $E_{7}$ Symmetric Area of the Black Hole Horizon, Phys. Rev. D 53 (1996) 5344 [hep-th/9602014] [SPIRES]. 